Portland State University

PDXScholar

Spring 2020

\title{
Scanning Electron Microscopy (SEM) Investigation of Morphology Changes in the Reduction of Silica Nanoparticles to Elemental Silicon
}

\author{
Allison M. Cairns \\ Portland State University
}

Follow this and additional works at: https://pdxscholar.library.pdx.edu/honorstheses

Part of the Inorganic Chemistry Commons, and the Nanoscience and Nanotechnology Commons Let us know how access to this document benefits you.

Recommended Citation

Cairns, Allison M., "Scanning Electron Microscopy (SEM) Investigation of Morphology Changes in the Reduction of Silica Nanoparticles to Elemental Silicon" (2020). University Honors Theses. Paper 919. https://doi.org/10.15760/honors.941

This Thesis is brought to you for free and open access. It has been accepted for inclusion in University Honors Theses by an authorized administrator of PDXScholar. Please contact us if we can make this document more accessible: pdxscholar@pdx.edu. 
Scanning Electron Microscopy (SEM) Investigation of Morphology Changes in the Reduction of Silica Nanoparticles to Elemental Silicon

by

\begin{abstract}
Allison Cairns
An undergraduate honors thesis submitted in partial fulfillment of the

Requirements for the degree of

Bachelor of Science

in

University Honors

and

Physics
\end{abstract}

Thesis Adviser

Dr. Andrea Goforth

Portland State University

2020 


\begin{abstract}
The application of silicon nanoparticles varies from energy storage materials, to drugdelivery, and molecular recognition. Various chemical and physical properties of the Si nanoparticles arise from their morphology. This paper aims to reveal the morphology of Si nanoparticles following magnesiothermic reduction of silica $\left(\mathrm{SiO}_{2}\right)$ nanoparticles. Two sets of $\mathrm{SiO}_{2}$ nanoparticles were used, commercially available NanoXact nanoparticles and laboratorysynthesized Stöber nanoparticles. A Zeiss Sigma VP FEG SEM was used to examine the morphology. Following the magnesiothermic reduction, the nanoparticles were etched with HF. Ten sets of images were taken of both Stöber and NanoXact nanoparticles: 1,2: the $\mathrm{SiO}_{2}$ nanoparticle starting materials; 3,4: the products after magnesiothermic reduction using $\mathrm{NaCl}$ as a heat sink; 5,6: the products after magnesiothermic reduction without $\mathrm{NaCl}$ as a heat sink; 7,8: the products after $\mathrm{HF}$ etching following magnesiothermic reduction with $\mathrm{NaCl} ; 9,10$ : the products after the HF etching following magnesiothermic reduction without $\mathrm{NaCl}$. This thesis is about the morphology changes in the reduction of silica nanoparticles to elemental silicon experiment. The experiment was conducted with the aim of achieving morphology preserving, reduced silicon nanoparticles for applications in various branches of science such as medical, materials, and chemistry.
\end{abstract}


TABLE OF CONTENTS

Cover Page

Abstract

List of Figures

1. Introduction

2. Experimental Section

a. Materials

b. Synthesis of Hollow Mesoporous Nanoparticles

c. XRD of the Nanoparticles

c. SEM Imaging of the Nanoparticles

3. Results
a. NanoXact
b. NanoXact with $\mathrm{NaCl}$
c. NanoXact without $\mathrm{NaCl}$
d. NanoXact with $\mathrm{NaCl}$ after $\mathrm{HF}$
e. b. NanoXact without $\mathrm{NaCl}$ after $\mathrm{HF}$
f. Stöber
g. Stöber without $\mathrm{NaCl}$
h. Stöber without $\mathrm{NaCl}$
i. Stöber with $\mathrm{NaCl}$ after $\mathrm{HF}$
j. Stöber without $\mathrm{NaCl}$ after $\mathrm{HF}$

4. Discussion

5. Conclusions

6. Future Work

Acknowledgments

References
PAGE

1

2

$4-7$

$8-12$

$12-21$

12

$12-15$

$16-17$

$17-21$

$21-50$

$21-22$

$24-26$

$27-28$

$29-30$

$30-32$

$33-34$

$35-36$

$37-39$

$40-44$

$45-50$

51

51-52

52

53

$54-55$ 


\section{LIST OF FIGURES}

Figure 1.1. Magnesiothermic reduction in a tube furnace.

Figure 1.2. Reaction Scheme for the magnesiothermic reduction process to produce silicon from silica, showing composition and morphology changes during the reaction.

Figure 1.3. X-ray diffractometry data of silicon nanoparticles, taken by James Barnes.

Figure 2.1. Flow chart of the experimental procedure. Ovals represent nanoparticle sets that were imaged.

Figure 2.2 Bragg's Law.

Figure 2.3 Interaction volume and various signals emitted from a sample.

Figure 2.4. Schematic of the Zeiss Sigma VP FEG SEM.

Figure 3.1. SEM micrographs of NanoXact nanoparticles imaged with the InLens detector and EHT of $10 \mathrm{kV}$. Left: 595 x magnification, scale bar: $10 \mathrm{um}$. Right: $20 \mathrm{kx}$ magnification, scale bar: $1 \mathrm{um}$.

Figure 3.2. Low magnification SEM micrographs of NanoXact nanoparticles imaged with an EHT of $10 \mathrm{kV}$, magnification of $595 \mathrm{x}$, and a $10 \mathrm{um}$ scale bar. Left: is imaged with the InLens detector. Right: is imaged with the SE2 detector.

Figure 3.3. High magnification SEM micrograph of NanoXact nanoparticles imaged with the InLens detector, EHT of $10 \mathrm{kV}, 150 \mathrm{kx}$ magnification, and a scale bar of $100 \mathrm{~nm}$.

Figure 3.4. High magnification SEM micrograph of NanoXact with $\mathrm{NaCl}$ nanoparticles imaged with the InLens detector, EHT of $5 \mathrm{kV}, 133.79 \mathrm{kx}$ magnification, and a scale bar of $100 \mathrm{~nm}$.

Figure 3.5. Low magnification SEM micrographs of NanoXact with $\mathrm{NaCl}$ nanoparticles, EHT of $5 \mathrm{kV}$, and a $27.79 \mathrm{kx}$ magnification. Left: InLens detector and a scale bar of $1 \mathrm{um}$. Right: SE2 detector and a scale bar of $1 \mathrm{um}$.

Figure 3.6. SEM micrographs of NanoXact with $\mathrm{NaCl}$ nanoparticles imaged at the same location with EHT of $5 \mathrm{kV}$. Top: lower magnification micrograph, $73.09 \mathrm{kx}$; scale bar of $300 \mathrm{~nm}$. Bottom: higher magnification micrograph, $217.75 \mathrm{kx}$; scale bar of $100 \mathrm{~nm}$.

Figure 3.7. Low magnification SEM micrograph of NanoXact nanoparticles without $\mathrm{NaCl}$, EHT of $5 \mathrm{kV}$, InLens detector, a $20 \mathrm{kx}$ magnification, and a $1 \mathrm{um}$ scale bar.

Figure 3.8. SEM micrographs of NanoXact nanoparticles without $\mathrm{NaCl}$, EHT of $5 \mathrm{kV}$, a scale bar of $100 \mathrm{~nm}$, and InLens detector. Left: $70 \mathrm{kx}$ magnification. Right: $150 \mathrm{kx}$ magnification.

Figure 3.9. Series of SEM micrographs of NanoXact nanoparticles without $\mathrm{NaCl}$. Imaged at the same location with EHT of $5 \mathrm{kV}$, varying magnification, and both detectors. Images on the left are imaged with the InLens detector; images on the right are imaged with the SE2 detector. 
Figure 3.10. Series of SEM micrographs of NanoXact nanoparticles with $\mathrm{NaCl}$ after the HF etching process imaged at EHT of $2.55 \mathrm{kV}$. Top micrographs were imaged at the same location with $20 \mathrm{kx}$ magnification and a scale bar of $1 \mathrm{um}$. The top left was imaged with the InLens detector, and the right was imaged with the SE2 detector. The bottom micrograph was imaged at $70 \mathrm{kx}$ and a scale bar of $100 \mathrm{~nm}$ with the InLens detector.

Figure 3.11. Series of SEM micrographs of NanoXact nanoparticles without $\mathrm{NaCl}$ after the HF etching process imaged at EHT of $2.55 \mathrm{kV}$ with the InLens detector. The top left was imaged with $20 \mathrm{kx}$, and a scale bar of $1 \mathrm{um}$. The top right was imaged at $70 \mathrm{kx}$ with a $100 \mathrm{~nm}$ scale bar. The following images were imaged at $150 \mathrm{kx}, 275 \mathrm{kx}$, and $300 \mathrm{kx}$ with scale bars of $100 \mathrm{~nm}, 100$ $\mathrm{nm}$, and $30 \mathrm{~nm}$, respectively.

Figure 3.12. Series of SEM micrographs of NanoXact nanoparticles with $\mathrm{NaCl}$ after the $\mathrm{HF}$ etching process imaged at EHT of $2.55 \mathrm{kV}$ with the InLens detector. The top left micrograph was imaged at $1.75 \mathrm{kx}$, and a scale bar of $10 \mathrm{um}$. The following images have a scale bar of $100 \mathrm{~nm}$. The top right was imaged with $70 \mathrm{kx}$ magnification. The bottom left micrograph was imaged at $150 \mathrm{kx}$, and the bottom right was imaged at $275 \mathrm{kx}$ magnification.

Figure 3.13. Series of SEM micrographs of Stöber nanoparticles imaged at EHT of $10 \mathrm{kV}$ with the InLens detector. The top micrograph was imaged at $2.81 \mathrm{kx}$ and a scale bar of $3 \mathrm{um}$. The bottom left micrograph was imaged at $20 \mathrm{kx}$ and a scale bar of $1 \mathrm{um}$. The bottom right was imaged at $75 \mathrm{kx}$ magnification and a scale bar of $100 \mathrm{~nm}$.

Figure 3.14. Series of SEM micrographs of Stöber nanoparticles imaged at EHT of $10 \mathrm{kV}$. The top micrographs were imaged at the same location, $20 \mathrm{kx}$ magnification, and a scale bar of $1 \mathrm{um}$. The top left was imaged with the InLens detector, and the top right was imaged with the SE2 detector. The bottom micrograph was imaged at $151.57 \mathrm{kx}$ and a scale bar of $100 \mathrm{~nm}$ with the SE2 detector.

Figure 3.15. SEM micrographs of Stöber nanoparticles with $\mathrm{NaCl}$ imaged at EHT of $5 \mathrm{kV}$ with the InLens detector. The top micrograph was imaged at $20 \mathrm{kx}$ and has a scale bar of $1 \mathrm{um}$. The bottom micrograph was imaged at $150 \mathrm{kx}$ and has a scale bar of $100 \mathrm{~nm}$.

Figure 3.16. SEM micrographs of Stöber nanoparticles with $\mathrm{NaCl}$ imaged at EHT of $5 \mathrm{kV}$. The top micrographs were imaged at the same location, have a scale bar of $1 \mathrm{um}$, and $20 \mathrm{kx}$ magnification. The bottom micrographs were imaged at the same location, with $186.60 \mathrm{kx}$ and a scale bar of $100 \mathrm{~nm}$. Images on the left: InLens, images on the right: SE2.

Figure 3.17. SEM micrographs of Stöber nanoparticles without $\mathrm{NaCl}$ imaged at EHT of $5 \mathrm{kV}$ and the InLens detector at the same location with varying magnification. The magnification is $8.75 \mathrm{kx}, 20 \mathrm{kx}, 70 \mathrm{kx}$, and $150 \mathrm{kx}$ with a scale bar of $1 \mathrm{um}, 1 \mathrm{um}, 100 \mathrm{~nm}$, and 100nm, respectively.

Figure 3.18. SEM micrographs of Stöber nanoparticles without $\mathrm{NaCl}$ imaged at EHT of $5 \mathrm{kV}$ and the InLens detector at the same location, scale bar of $100 \mathrm{~nm}$, and $150 \mathrm{kx}$ magnification.

Figure 3.19. SEM micrographs of Stöber nanoparticles without $\mathrm{NaCl}$ imaged at EHT of $5 \mathrm{kV}$ at the same location with $20 \mathrm{kx}$ magnification and a scale bar of $1 \mathrm{um}$. Top: InLens detector.

Bottom: SE2 detector. 
Figure 3.20. SEM micrographs of Stöber nanoparticles with $\mathrm{NaCl}$ after being etched with $\mathrm{HF}$ imaged at EHT of $2.55 \mathrm{kV}$ at the same location. From top left to right, the magnification was 200 $\mathrm{kx}, 300 \mathrm{kx}$, and $300 \mathrm{kx}$; the scale bars are $100 \mathrm{~nm}, 30 \mathrm{~nm}$, and $30 \mathrm{~nm}$. Left: InLens detector. Right: SE2 detector.

Figure 3.21. SEM micrographs of Stöber nanoparticles with $\mathrm{NaCl}$ after being etched with $\mathrm{HF}$ imaged at EHT of $2.55 \mathrm{kV}$ at the same location with the InLens detector. Top left: $20 \mathrm{kx}$ with a scale bar of $1 \mathrm{um}$. Top right: $70 \mathrm{kx}$, scale bar of $100 \mathrm{~nm}$. Bottom: $150 \mathrm{kx}$, scale bar of $100 \mathrm{~nm}$.

Figure 3.22. SEM micrograph of Stöber nanoparticles with $\mathrm{NaCl}$ after being etched with $\mathrm{HF}$ imaged at EHT of $2.55 \mathrm{kV}$ with the InLens detector, a magnification of $1.00 \mathrm{kx}$, and scale bar of $10 \mathrm{um}$.

Figure 3.23. SEM micrograph of Stöber nanoparticles with $\mathrm{NaCl}$ after being etched with $\mathrm{HF}$ imaged at EHT of $2.55 \mathrm{kV}$ with the InLens detector. Top left: $20 \mathrm{kx}$ magnification and a scale bar of $1 \mathrm{um}$. The following images have a scale bar of $100 \mathrm{~nm}$. Top right: $70 \mathrm{kx}$ magnification. Bottom left: $150 \mathrm{kx}$ magnification. Bottom right: $275 \mathrm{kx}$ magnification.

Figure 3.24. SEM micrograph of Stöber nanoparticles with $\mathrm{NaCl}$ after being etched with $\mathrm{HF}$ imaged at EHT of $2.55 \mathrm{kV}$, with a scale bar of $100 \mathrm{~nm}$, and imaged with the InLens detector. Top: $178.44 \mathrm{kx}$ magnification. Bottom: $275 \mathrm{kx}$ magnification.

Figure 3.25. SEM micrograph of Stöber nanoparticles without $\mathrm{NaCl}$ after being etched with $\mathrm{HF}$ imaged at EHT of $2.55 \mathrm{kV}$ with the InLens detector, a scale bar of $1 \mathrm{um}$, and $20 \mathrm{kx}$ magnification.

Figure 3.26. SEM micrograph of Stöber nanoparticles without $\mathrm{NaCl}$ after being etched with $\mathrm{HF}$ imaged at EHT of $2.55 \mathrm{kV}$ with the InLens detector at the same location. Top left: $20 \mathrm{kx}$ magnification and a scale bar of $1 \mathrm{um}$. Top right: $70 \mathrm{kx}$ magnification and a scale bar of $100 \mathrm{~nm}$. Bottom left: $150 \mathrm{kx}$ magnification and a scale bar of $100 \mathrm{~nm}$. Bottom right: $275 \mathrm{kx}$ magnification and a scale bar of $30 \mathrm{~nm}$.

Figure 3.27. SEM micrograph of Stöber nanoparticles without $\mathrm{NaCl}$ after being etched with $\mathrm{HF}$ imaged at EHT of $2.55 \mathrm{kV}$ with the InLens detector at the same location. Top left: $20 \mathrm{kx}$ magnification and a scale bar of $1 \mathrm{um}$. Top right: $70 \mathrm{kx}$ magnification and a scale bar of $100 \mathrm{~nm}$. Bottom left: $150 \mathrm{kx}$ magnification and a scale bar of $100 \mathrm{~nm}$. Bottom right: $300 \mathrm{kx}$ magnification and a scale bar of $30 \mathrm{~nm}$.

Figure 3.28. SEM micrograph of Stöber nanoparticles without $\mathrm{NaCl}$ after being etched with $\mathrm{HF}$ imaged at EHT of $2.55 \mathrm{kV}$ at the same location, $20 \mathrm{kx}$, and $1 \mathrm{um}$ scale bar. Top: InLens detector. Bottom: SE2 detector.

Figure 3.29. SEM micrograph of Stöber nanoparticles without $\mathrm{NaCl}$ after being etched with $\mathrm{HF}$ imaged at EHT of $2.55 \mathrm{kV}$ at the same location. All of the images on the left: InLens. All images on the right: SE2. Top images: $8 \mathrm{kx}$ magnification, 1 um scale bar. The following images have a $100 \mathrm{~nm}$ scale bar. Middle images: $70 \mathrm{kx}$ magnification. Bottom images: $150 \mathrm{kx}$ magnification. 
Figure 3.30. SEM micrograph of Stöber nanoparticles without $\mathrm{NaCl}$ after being etched with $\mathrm{HF}$ imaged at EHT of $2.55 \mathrm{kV}$ with the InLens detector, a $100 \mathrm{~nm}$ scale bar, and $275 \mathrm{kx}$ magnification.

Table 3.1 Summary of experimental results. 


\section{INTRODUCTION}

Si nanoparticles are commonly used in applications that involve charge storage, molecular recognition, or controllable NO release. ${ }^{1,2}$ With its diverse set of applications, the synthesis of size and morphology-controlled Si nanoparticles could implement improvements across various branches of science. Si nanoparticles come in both dense crystalline and porous forms. Porous silicon may prove to be an excellent nanomaterial for lithium-ion battery anodes, because it may simultaneously address the poor cyclability and low rates of bulk phase Si anodes by providing smaller silicon substructures for lithium diffusion and minimizing stresses. ${ }^{1}$ Comparatively, dense crystalline Si nanoparticles are more challenging to synthesize, especially with a high degree of crystallinity and morphology control. The overarching goal of this project is to synthesize dense, crystalline Si nanostructures that can be used in several areas of science, such as the applications previously mentioned.

Porous silicon can be synthesized from silica by magnesiothermic reduction and contains smaller, dense crystalline silicon domains. The silica starting material is an amorphous network of silicon and oxygen. Magnesium is considered an effective reducing agent for silica reduction according to equation 1 ; however, it also causes morphology change and structural damage. ${ }^{3}$

$$
\mathrm{SiO}_{2}+2 \mathrm{Mg} \rightarrow \mathrm{Si}+2 \mathrm{MgO}
$$

Previous research shows that approximately one-quarter of the precursor silicon, silica $\left(\mathrm{SiO}_{2}\right)$ is converted into elemental silicon $(\mathrm{Si})$ while the remaining byproduct is silica $\left(\mathrm{SiO}_{2}\right){ }^{7}$ Magnesiothermic reduction is one of the most commonly used techniques for reducing silica to silicon nanostructures, it occurs at $650^{\circ} \mathrm{C}$ where silica and magnesium form silicon and magnesium oxide. 


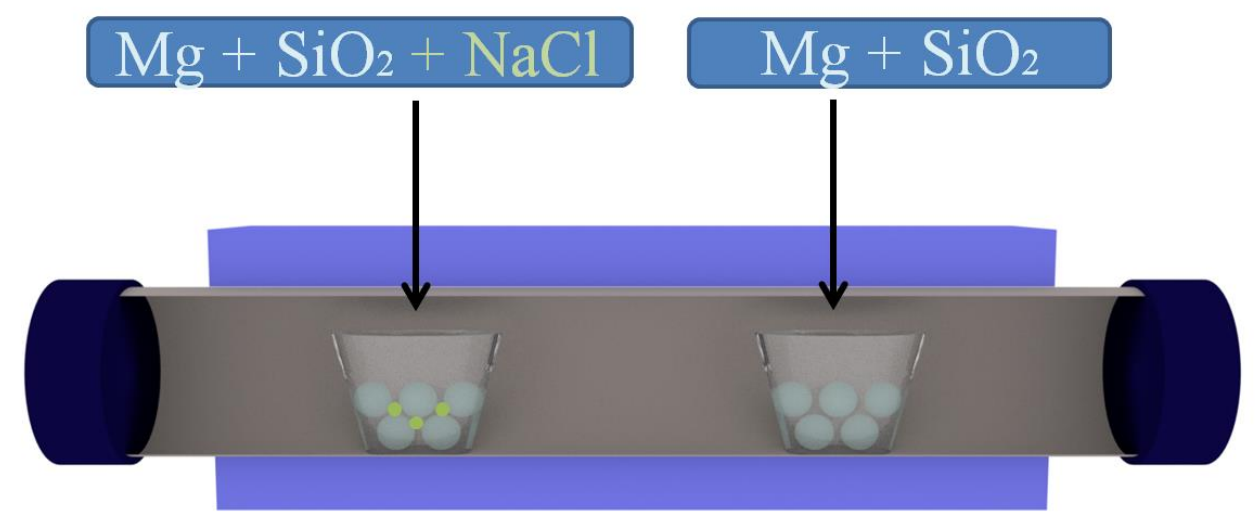

\section{$650^{\circ} \mathrm{C}$}

Figure 1.1. Magnesiothermic reduction in a tube furnace.

The reaction takes place in a high-temperature tube furnace that has air-free manipulation, as displayed in Figure 1.1.
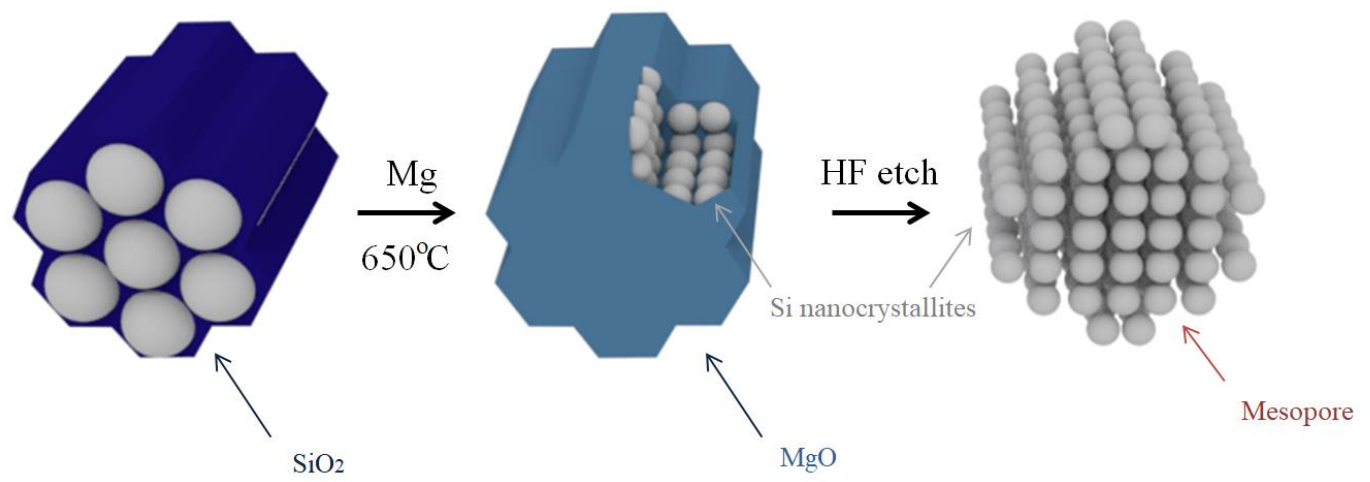

Figure 1.2. Reaction Scheme for the magnesiothermic reduction process to produce silicon from silica, showing composition and morphology changes during the reaction. 
Even when using a nonporous feedstock, magnesiothermic reduction of silica to silicon offers a scalable and straightforward choice for the creation of porous silicon structures (see Figure 1.2). ${ }^{1} \mathrm{SiO}_{2}$ has an amorphous structure; following magnesiothermic reduction, silica reduces to $\mathrm{Si}$ and $\mathrm{MgO}$, and $\mathrm{SiO}_{2}$ are produced as byproducts. After the sample is etched with $\mathrm{HF}$, a non-polar surface forms on the $\mathrm{Si}$, producing $\mathrm{Si}$ spheres. This thesis aims to use the SEM to deduce what type of morphology silica has following magnesiothermic reduction as a function of the reaction conditions and after workup, to isolate morphologically uniform dense crystalline Si nanoparticles. As shown in Figure 1.2, we expected the nanoparticles to be contained within initially larger porous structures and accessible via an appropriate workup.

The nanoparticles in this experiment were characterized by scanning electron microscopy (SEM). The primary factors for achieving a high-quality scanning electron micrograph include a high accelerating voltage $(E H T)$, high signal to noise ratio $(S: N)$, and frame averaging $(N)$ for maximum resolution. To acquire a nominal signal to noise ratio, SEM parameters were adjusted. Varying SEM parameters included the accelerating voltage and detector type. The primary detectors used in these experiments were the InLens Duo detector, and the SE2 - Secondary Electron detector (Everhart-Thornley).

Stöber silica particles are usually nonporous and monodisperse spheres with colloidal range diameters. To achieve desired properties, in particular monodispersity, absence of porosity, aggregation free isolability, diameter, and spherical shape, the synthesis of Stöber silica particles as a basis for redox modification may be optimal. ${ }^{2}$ Variation in particle diameters occurs by altering concentrations of the reagents and reactants, the type of solvent, and temperature. ${ }^{2}$ An ideal temperature range in which a change in temperature has little or no effect on particle diameter during the reaction is used to achieve low polydispersity of nanoparticles. ${ }^{2}$ The 
overarching goal is to create silicon nanoparticles that are approximately $25 \mathrm{~nm}$ in diameter, with approximately $5 \mathrm{~nm}$ pores.

Previous work in The Goforth Lab done by James Barnes explains that monodisperse $\mathrm{SiO}_{2}$ particles can be used to prepare monodisperse Si particles. Figure 1.3 displays x-ray diffractometry (XRD) results of laboratory synthesized silicon (Si) nanoparticles.

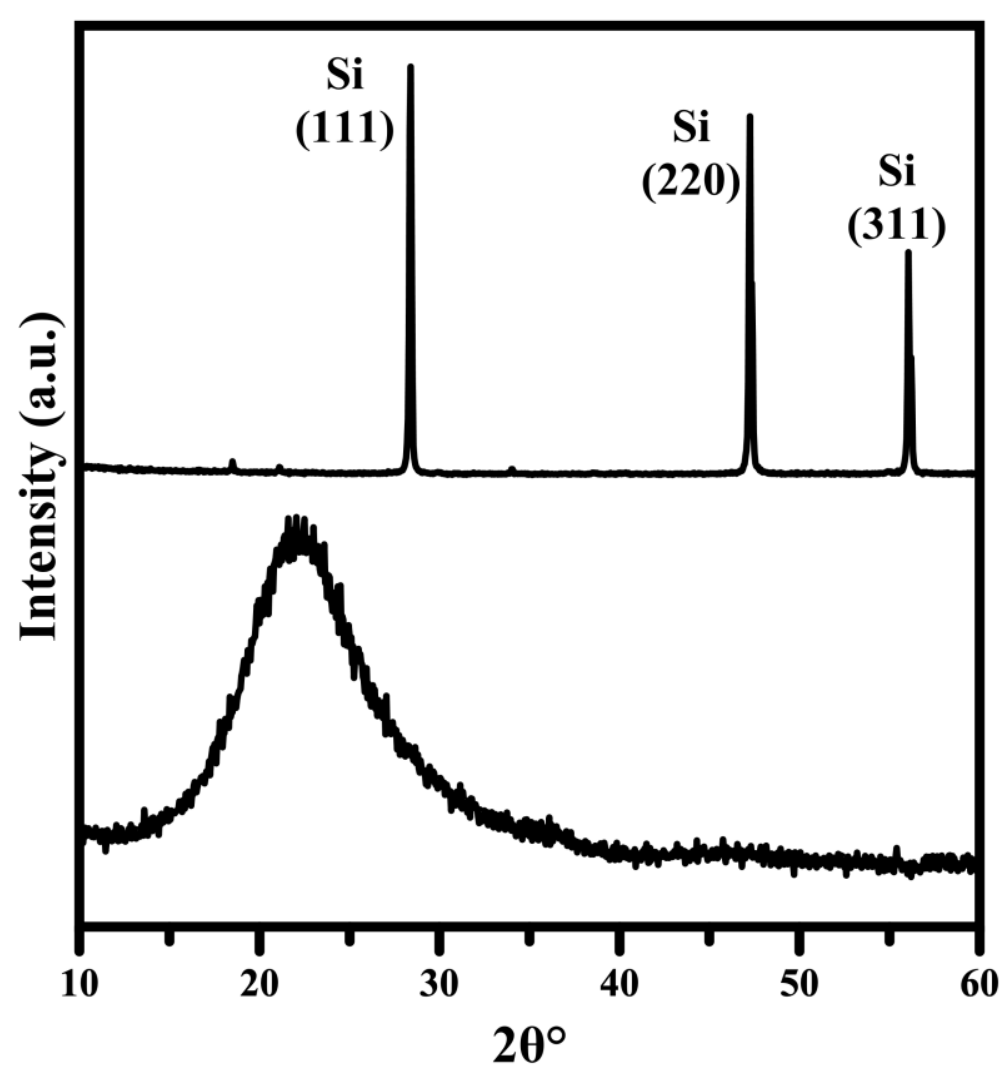

Figure 1.3. X-ray diffractometry data of silicon nanoparticles, taken by James Barnes.

The XRD data from James Barnes' Si samples had a mismatch, which led the group to believe the morphology of the nanoparticles varied. To determine the morphology of the elemental silicon nanoparticles, a Scanning Electron Microscope (SEM) was used since SEMs produce high-resolution three-dimensional-like images. Various reaction conditions were altered, 
such as the addition of $\mathrm{NaCl}$ as a heat sink in magnesiothermic reduction and $\mathrm{HCl}$ to etch remaining silica $\left(\mathrm{SiO}_{2}\right)$ after magnesiothermic reduction.

\section{EXPERIMENTAL}

\section{Materials:}

The reagents and materials used for this experiment are absolute ethanol (Abs Eth), ammonium hydroxide $\left(\mathrm{NH}_{4} \mathrm{OH}\right)$, tetraethyl orthosilicate (TEOS), triethylamine (TEA), cetyl trimethylammonium bromide (CTAB), anhydrous sodium carbonate (NaCO3), deionized water (DI H2O), 1-neck round bottom flasks (50 mL, $100 \mathrm{~mL}$, and $250 \mathrm{~mL}), 10 \mathrm{~mL}$ separatory funnel, vacuum flask, and a hot plate for stirring (max $1200 \mathrm{rpm})$ and temperature control.

NanoXact nanoparticles are created by the company nanoComposix. For this work, 80 nm dried Silica Nanospheres were used alongside silica nanoparticles from the lab.

Synthesis of Hollow Mesoporous Nanoparticles:

The following procedure was adapted from, ${ }^{4}$ Hadipour Moghddam, S. P.; Yazdimamaghani, M.; Ghandehari, H. Glutathione-Sensitive Hollow Mesoporous Silica Nanoparticles for Controlled Drug Delivery. J. Control. Release 2018 with modifications. Figure 2.1 represents the steps in the procedure. Before magnesiothermic reduction, commercially available NanoXact silica particles and lab synthesized Stöber nanoparticles were imaged. The respective nanoparticles underwent magnesiothermic reduction with and without the addition of $\mathrm{NaCl}$ as a heat sink. The resulting four sets of silicon nanoparticles were imaged. Due to silica impurities in the elemental silicon nanoparticles, the previously mentioned four sets of nanoparticles were then etched with HF. The etching process should produce elemental Si 
whether or not $\mathrm{NaCl}$ was used as a heat sink. This would result in the morphology of reduced silicon. The nanoparticles were then imaged after the HF etching.

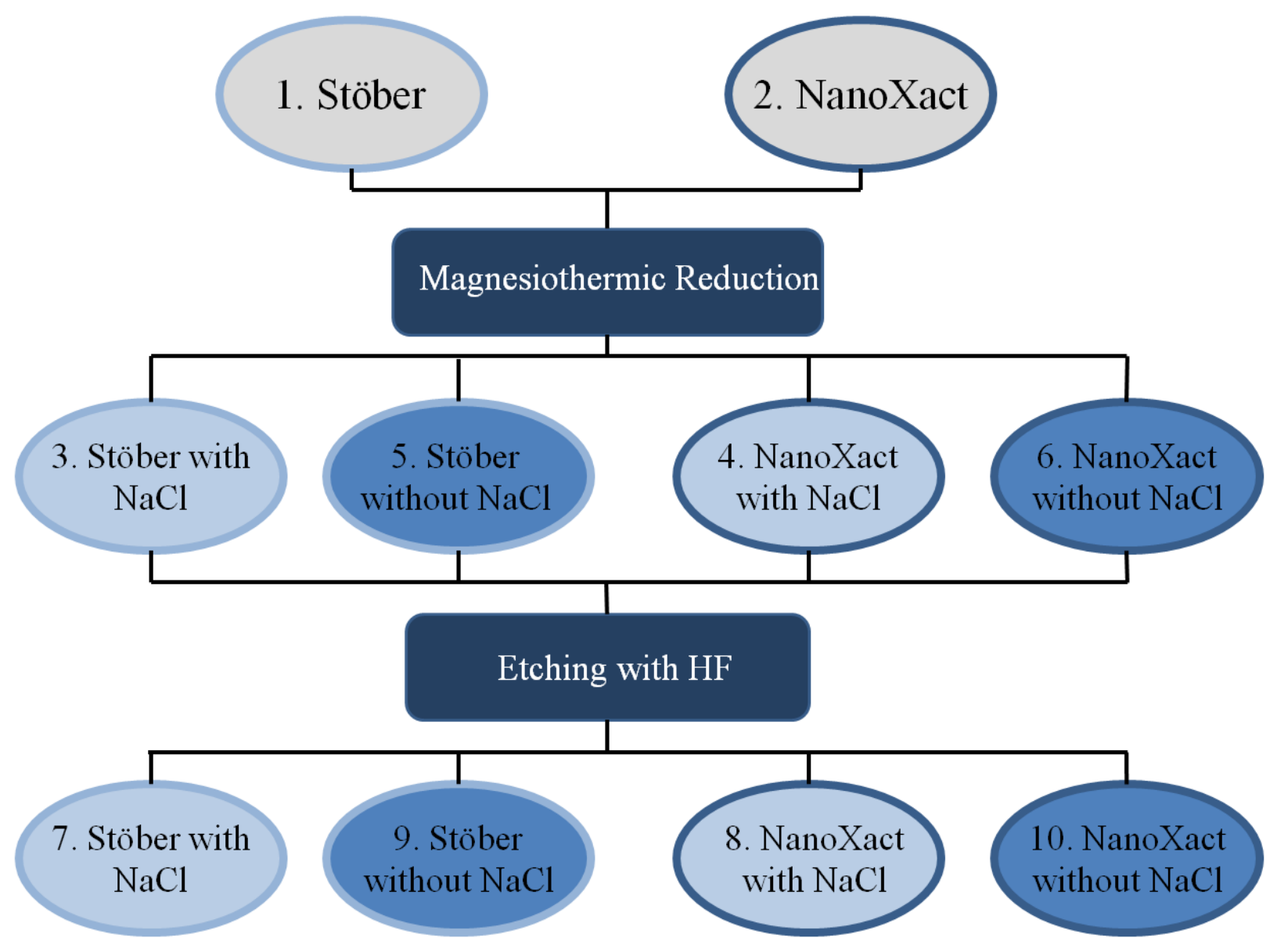

Figure 2.1. Flow chart of the experimental procedure. Ovals represent nanoparticle sets that were imaged.

A magnetic stirrer, $99 \mathrm{~mL}$ of $\mathrm{ABS}$ Eth, $3.24 \mathrm{~mL} \mathrm{DI} \mathrm{H2O}$, and $7.8 \mathrm{~mL} \mathrm{NH}_{4} \mathrm{OH}$ was stirred in a $250 \mathrm{~mL}$ round bottom flask for $10 \mathrm{~min}$ at $400 \mathrm{rpm}$ at room temperature. $4 \mathrm{~mL}$ of TEOS was added dropwise using a separation funnel for $25 \mathrm{~min}$. The mixture was left to stir overnight. 
The second day, the particles were cooled and centrifuged. The nanoparticles were spun down and suspended in $70 \%$ ethanol. Following the suspension, the washing procedure was repeated two more times. The nanoparticles were oven-dried at $60^{\circ} \mathrm{C}$ overnight.

$180 \mathrm{mg}$ of the dried nanoparticles were placed into a $200 \mathrm{~mL} \mathrm{RBF}$, and $33.4 \mathrm{~mL}$ of DI $\mathrm{H} 2 \mathrm{O}$ was added. The particles were thoroughly sonicated. $3.5 \mathrm{~mL}$ of Abs Et, and $28 \mu \mathrm{L}$ of TEA and $0.06 \mathrm{~g}$ of CTAB were added. The mixture was stirred on a hot plate for $50 \mathrm{~min}$ at $600 \mathrm{rpm}$ at $80^{\circ} \mathrm{C} .144 \mu \mathrm{L}$ TEOS was added all at once, then the stir rate was increased to $1200 \mathrm{rpm}$ for 4 hours at $80^{\circ} \mathrm{C}$. Once cooled, the nanoparticles were spun down and washed twice with $70 \%$ ethanol and once with DI H2O.

On the third day, the nanoparticles underwent magnesiothermic reduction. The silica was weighed in grams and converted to moles, then the amount of magnesium necessary was calculated using equation 2 .

$$
\mathrm{SiO}_{2} \text { grams }\left(\frac{1 \mathrm{~mol}}{60 \mathrm{~g} \text { silica }}\right)=\text { moles } \mathrm{SiO}_{2}
$$

Equation 1 shows the 1:2 ratio from the magnesiothermic reduction of silica to silicon. Multiplying the moles silica by two results in the moles magnesium needed. Equation 3 is used to convert moles $\mathrm{Mg}$ to grams.

$$
\text { moles } \operatorname{Mg}\left(\frac{24.3 \mathrm{~g} \mathrm{Mg}}{1 \mathrm{~mol}}\right)=\text { grams } \mathrm{Mg}
$$

$\mathrm{NaCl}$ was added as a heat sink to preserve morphology. The Thermo Scientific Lindberg/ Blue MTube furnace was used with a nitrogen tank. The samples started at $35^{\circ} \mathrm{C}$, and the temperature 
was increased at a rate of $20^{\circ} \mathrm{C}$ per minute, then set to $650^{\circ} \mathrm{C}$ for $5 \mathrm{hrs}$. The samples were then cooled for an hour until reaching $35^{\circ} \mathrm{C}$.

The following day the nanoparticles were suspended and thoroughly sonicated in $10 \mathrm{~mL}$ DI H2O. $1.33 \mathrm{~g}$ of $\mathrm{NaCO}_{3}$ was added to a $50 \mathrm{~mL}$ round bottom flask, $10 \mathrm{~mL} \mathrm{DI} \mathrm{H}_{2} \mathrm{O}$ was added and stirred on a hot plate for 30 minutes at $400 \mathrm{rpm}$ at $50^{\circ} \mathrm{C}$. Stirring was increased to $1200 \mathrm{rpm}$, and the nanoparticle suspension was added. The reaction proceeded for 10 hours.

The nanoparticles were then spun down in the centrifuge and washed twice with $70 \%$ ethanol and washed once with Abs Eth. $45 \mathrm{~mL}$ Abs Eth and $5 \mathrm{~mL} \mathrm{HCl}$ were mixed, and the washed nanoparticles were resuspended, then washed and sonicated thoroughly. The solution was refluxed at $80^{\circ} \mathrm{C}$ for 1 hour. Following the reflux, it was cooled down and washed three times with $70 \%$ Abs Eth, then left to dry overnight at $60^{\circ} \mathrm{C}$.

After imaging the nanoparticles following magnesiothermic reduction with and without $\mathrm{NaCl}$ as a heat sink, the nanoparticles were etched with $\mathrm{HF}$ to remove silica byproduct. A 1:1:1 ratio was used of $\mathrm{DI} \mathrm{H}_{2} \mathrm{O}$, ethanol, and $49 \%$ wt $\mathrm{HF}$. $\mathrm{HF}$ attacks the $\mathrm{SiO}$ bonds and leaves the $\mathrm{Si}$ surface with a hydride, $\mathrm{SiH}$, instead of $\mathrm{SiO}$, creating a non-polar surface. $5 \mathrm{~mL} \mathrm{DI}, 5 \mathrm{~mL}$ ethanol, and $5 \mathrm{~m} \mathrm{HF}$ were added to a tube with a stir bar. HF was added to the solution while stirring for 15 minutes; the solution was sonicated for 45 seconds, then toluene was added to the sample.

Characterization of nanoparticles is typically achieved with X-ray diffraction (XRD), neutron diffraction, and transmission electron microscopy, so that measurements can be made on crystallographic structures. XRD and SEM were used to characterize the nanoparticles in this experiment. 
XRD of the Nanoparticles:

In XRD, typically, powder diffraction is used; peaks are called reflections, which are linear superpositions in the crystal. There are two types of $\mathrm{x}$-rays: characteristic $\mathrm{x}$-rays and Bremsstrahlung x-rays. Characteristic x-rays occur when an electron collides with an inner shell electron, and both are ejected from the atom. An outer shell electron then falls into the inner shell, and a characteristic $\mathrm{x}$-ray is released. Bremsstrahlung $\mathrm{x}$-rays occur when an electron that passes near the nucleus is slowed down and deflected. An x-ray beam contains approximately $80 \%$ of Bremsstrahlung $x$-rays. The $x$-ray energy spectrum can be manipulated by changing current or voltage settings, or by adding filters. In XRD, a voltage is applied to the filament, which increases the current; this causes electrons to be accelerated on the negative cathode towards a tungsten (W) target anode. In turn, this causes the production of x-rays where a monochromatic wavelength $(\lambda)$ is used, and $x$-ray damage is negligible. When considering which atom is hit by the x-ray first, all atoms form spherical waves and excite the next atom. Fourier transforms are a mathematical model of diffraction of molecules where the complex numbers are in the amplitude and phase. When the wave function collapses by recording, it is no longer complex, but real.

Bragg's law states that an incident $\mathrm{x}$-ray will reflect with the same angle of scattering. A heuristic version of Bragg's Law is demonstrated in Figure 2.2. 


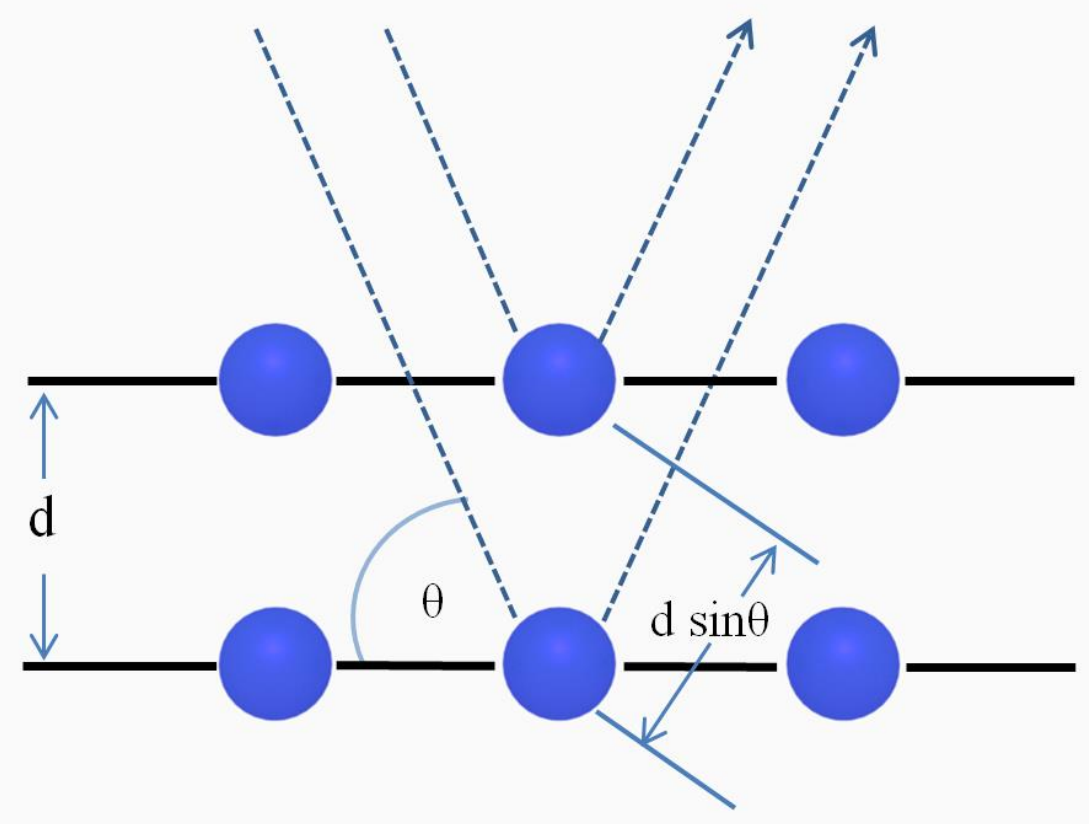

Figure 2.2 Bragg's Law.

Bragg's Law for X-Ray Diffraction equation 4, is displayed below:

$$
\mathrm{n} \lambda=2 \mathrm{~d} \sin (\theta)
$$

where $\mathrm{n}$ is an integer called the order of the diffracted beam, $\lambda$ is the wavelength of the incoming beam, $\mathrm{d}$ is the $\mathrm{d}$-spacing, and theta is the angle of incidence that equals the angle of reflection ${ }^{5}$.

\section{SEM Imaging of the Nanoparticles:}

Secondary electron microscopy gives information on the characterization of heterogeneous organic and inorganic material with nanometer resolution ${ }^{5}$. SEM micrographs produce three-dimensional-like images due to the SEM's large depth of field. The Zeiss Sigma VP FEG Scanning Electron Microscope (SEM), as displayed in Figure 2.2, is composed of a 
field emission gun (FEG), electromagnetic lens system, magnetic coils, electron detectors, and associated electronics. The FEG generates and accelerates an electron beam and can achieve an energy range up to $10^{4} \mathrm{KeV}$ range. Equation 5 details how electron wavelength can be calculated from the momentum of an electron accelerated from the FEG.

$$
\lambda=\sqrt{\frac{h^{2}}{2 m e V}}=\frac{1.23 \mathrm{~nm}}{\sqrt{V}}
$$

Where $\lambda$ is the electron wavelength, $\mathrm{V}$ is in volts, and $\mathrm{h}$ is Planck's constant. High voltages generate a larger interaction volume by penetrating farther into the sample. Equations 6 and 7 show that an accelerated electron through a potential $\mathrm{eV}$ has a kinetic energy of

$$
\frac{1}{2} \mathrm{mv}^{2}=\mathrm{eV}
$$

and a momentum of

$$
\mathrm{p}=\mathrm{mv}=\sqrt{2 m e V}
$$

The electromagnetic lens system is used to demagnify the spot size to produce a narrowfocused electron spot on the sample, which contains adequate probe current to form a highresolution micrograph. Equation 8 shows the Lorentz force equation, which propels the electron beam:

$$
\mathrm{F}=\mathrm{q}(\vec{v} \times \vec{B})
$$

where $\mathrm{F}$ is the magnetic force, $\mathrm{q}$ is the charge of an electron, $\vec{v}$ is velocity, and $\vec{B}$ is the magnetic field. Magnetic coils control the beam. The beam rasters over the sample to produce a two- 
dimensional image. The electron beam produced from the final lens interacts with the sample to generate signals used to form an image ${ }^{6}$. Figure 2.3 shows that electron beam interactions can emit specific signals from a specimen.

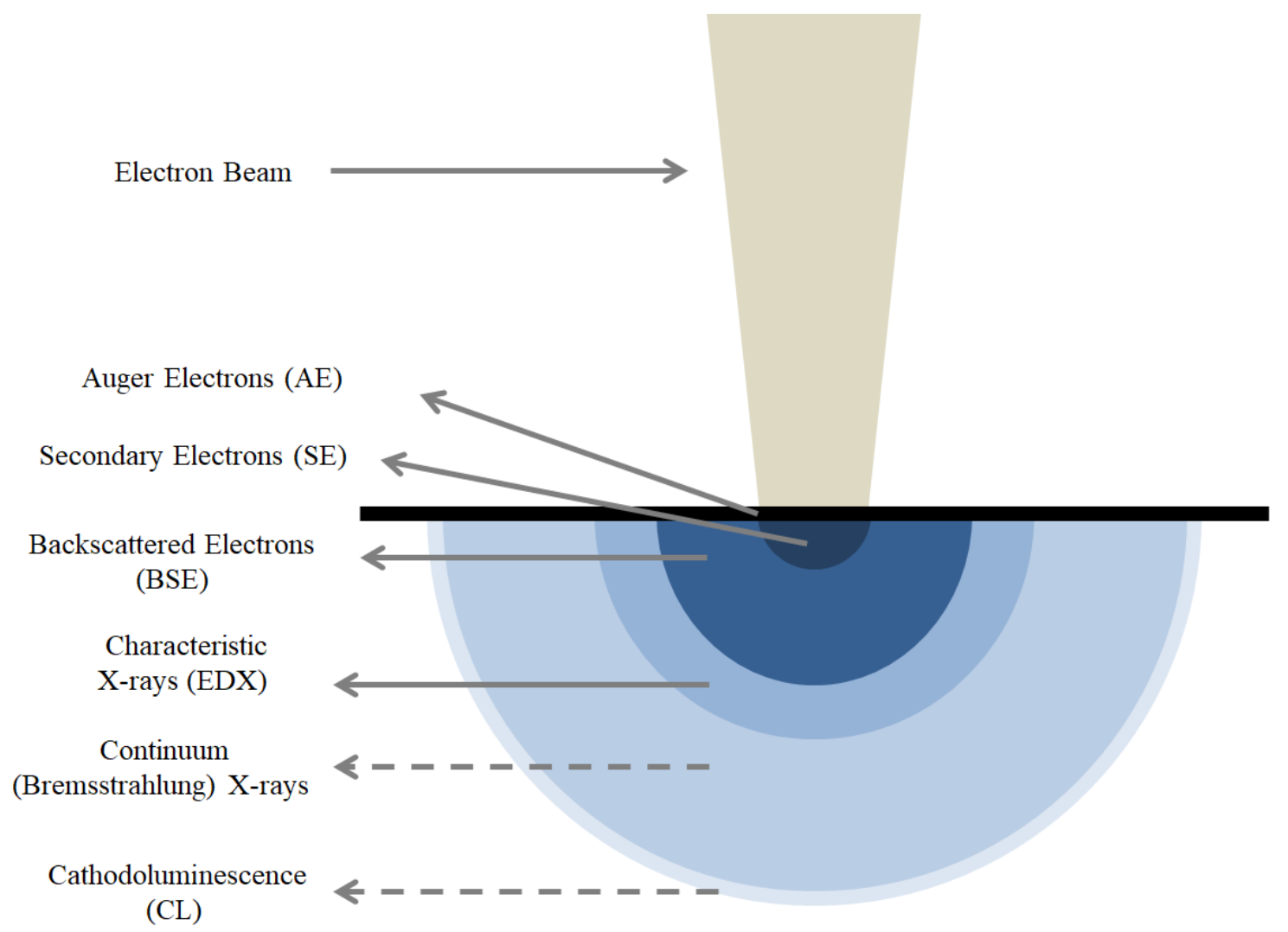

Figure 2.3 Interaction volume and various signals emitted from a sample.

Electron detectors vary from secondary electron detectors which relay topographic information; to energy-dispersive $\mathrm{x}$-ray spectroscopy detectors (EDX/ EDS) which interpret x-rays for elemental analysis of the sample; and backscatter detectors which display heavy elements (high $Z$ ) as white, and light typically organic elements in dark shades of grey. Various electronics are used to alter and regulate SEM conditions. Computers apply a second Fourier transform to produce an image. The maximum resolution imposed by the diffraction limit is shown in equation 9. 


$$
\mathrm{d}_{0}=\frac{0.61 \lambda}{n \sin \sin \alpha_{0}} \approx \frac{0.61 \lambda}{\alpha_{0}}
$$

Electrons have an $\mathrm{n}$ value $\sim 1$, and the angles are small, so $\mathrm{n} \sin \alpha_{0}$ approximates to $\alpha_{0}$. Resolution can be improved by a small aperture, short working distance (WD), and strong condenser lens.

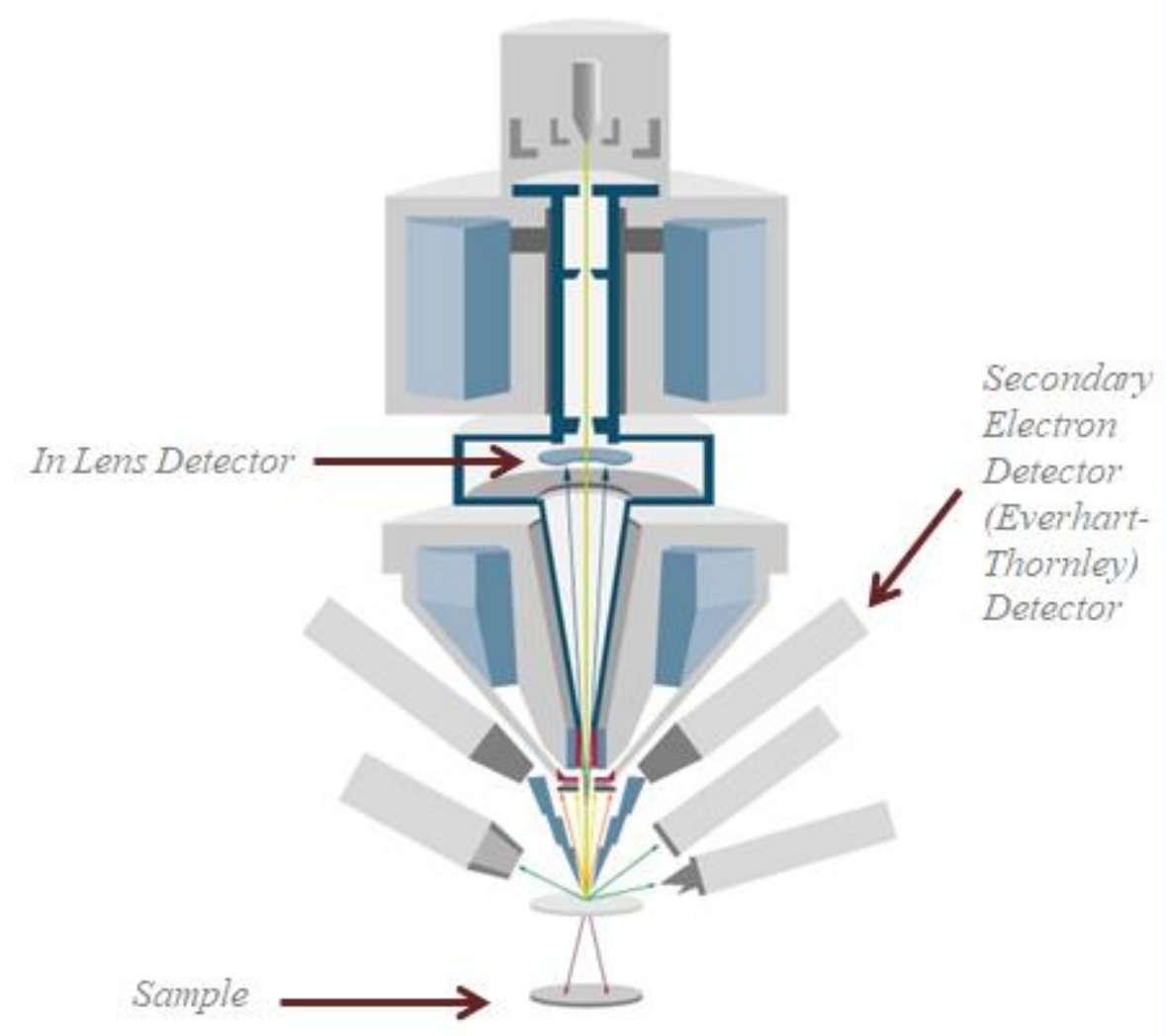

Figure 2.4. Schematic of the Zeiss Sigma VP FEG SEM.

The Zeiss Sigma VP FEG SEM is a complex configuration, as displayed in Figure 2.3. The following procedure is for imaging nanoparticles with the Zeiss Sigma VP FEG SEM, 
advice for imaging nanoparticles was given by Greg Baty of CEMN. A $30 \mu \mathrm{m}$ aperture was used without high current. The accelerating voltages used for imaging were $2.55 \mathrm{kV}, 5 \mathrm{kV}$, and $10 \mathrm{kV}$. Frame averaging varied from $N=4$ to $N=64$. The secondary electron detectors used were the InLens Duo detector and the SE2 - Secondary Electron (Everhart-Thornley) Detector.

\section{RESULTS}

\section{NanoXact:}

Figure 3.1 displays low magnification images of the commercially available NanoXact silica $\left(\mathrm{SiO}_{2}\right)$ nanoparticles prior to magnesiothermic reduction. The image on the left shows clusters of silica nanoparticles of varying shapes and sizes. When magnification is increased in the right image of Figure 3.1, spherical particles are identified, forming the large clusters on the left image of Figure 3.1.

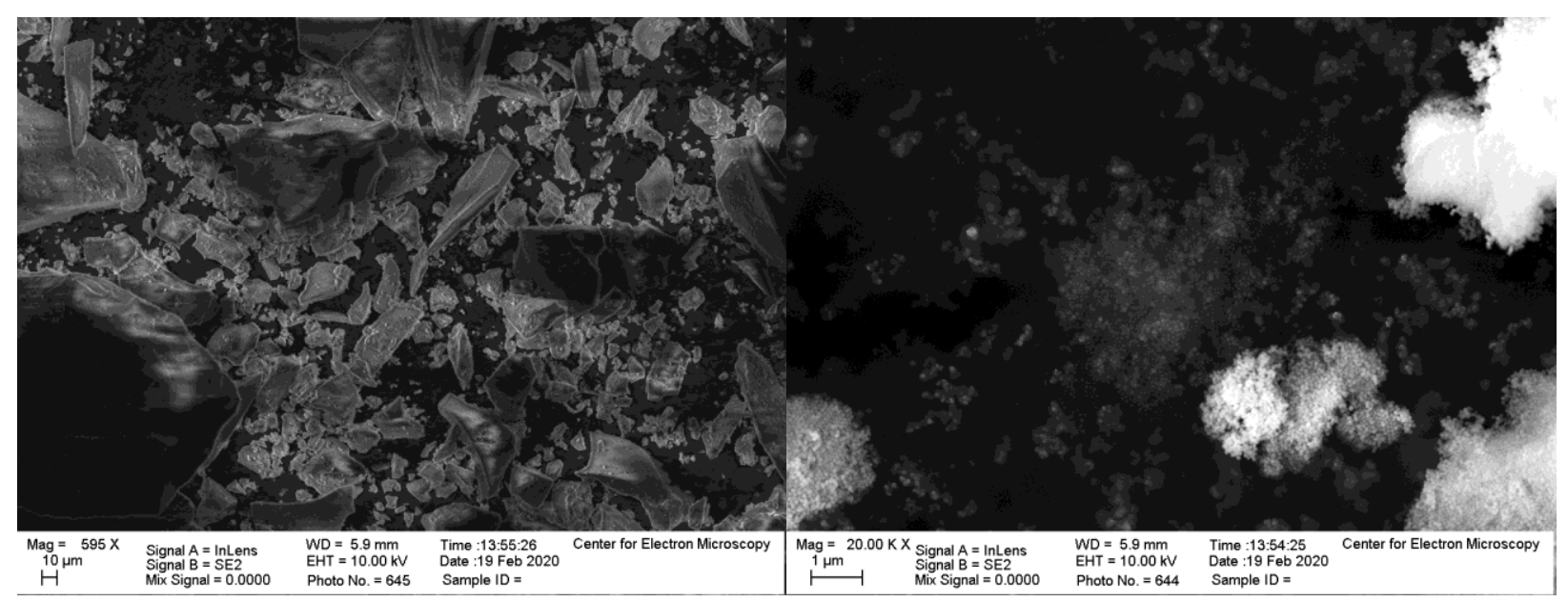

Figure 3.1. SEM micrographs of NanoXact nanoparticles imaged with the InLens detector and EHT of 10 kV. Left: 595 x magnification, scale bar: 10 um. Right: 20 kx magnification, scale bar: 1 um. 
The InLens detector has a higher signal to noise ratio $(\mathrm{S}: \mathrm{N})$ in comparison to the secondary electron detector. A high $\mathrm{S}: \mathrm{N}$ is seen in an image that is clear in comparison to grainy. Since the InLens detector is located normal $\left(90^{\circ}\right)$ to the face of the sample, topographic detail is lost in the images, producing a morphology that appears to be flattened. The secondary electron detector (SE2) has a lower S:N creating grainy images in comparison to the InLens detector. The SE2 detector shows greater topographic detail due to its location in the SEM, which results in greater morphological detail in the images. Figure 3.2 illustrates a side by side comparison of the detectors imaged in the same location and magnification.

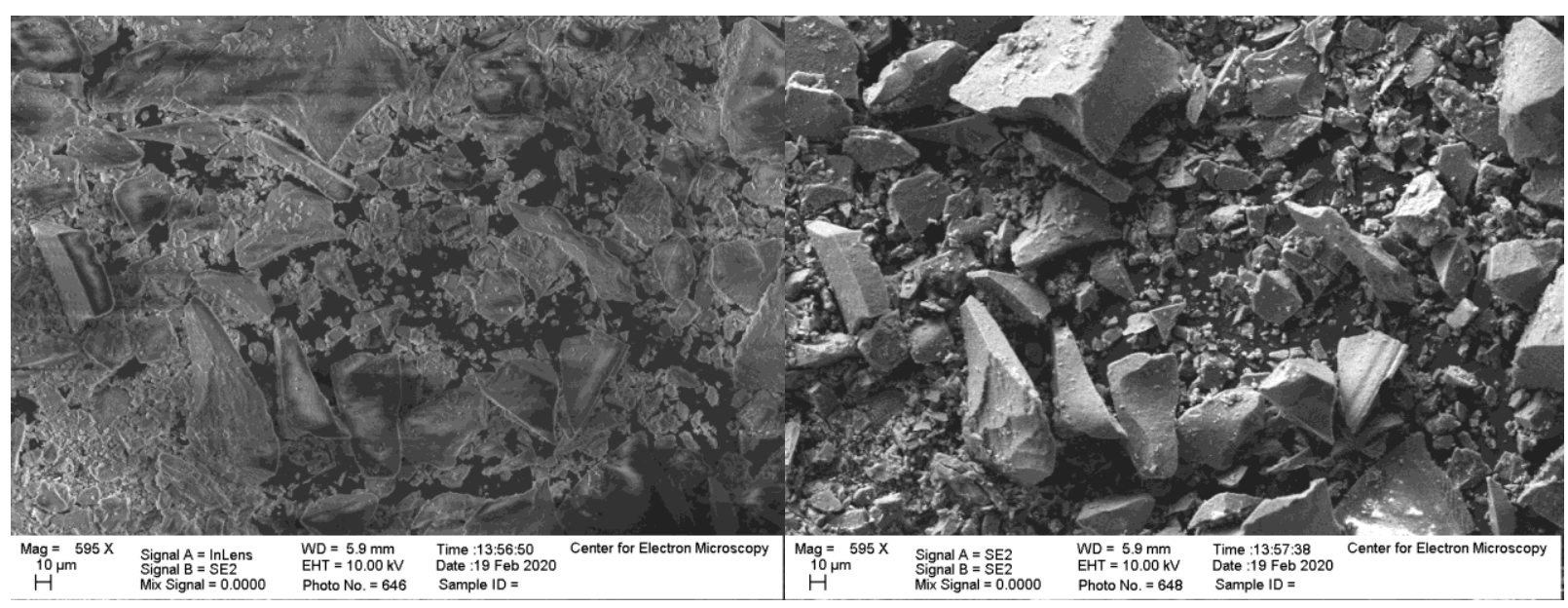

Figure 3.2. Low magnification SEM micrographs of NanoXact nanoparticles imaged with an EHT of $10 \mathrm{kV}$, magnification of $595 \mathrm{x}$, and a 10 um scale bar. Left: is imaged with the InLens detector. Right: is imaged with the SE2 detector.

At low magnification, the NanoXact silica nanoparticle clusters have a nonuniform size distribution. Additionally, the shape of the clusters varies as well; some clusters appear to have flat faces while others have curved faces. The amorphous shape of the silica clusters is due to the short-range order of its crystallographic structure. 


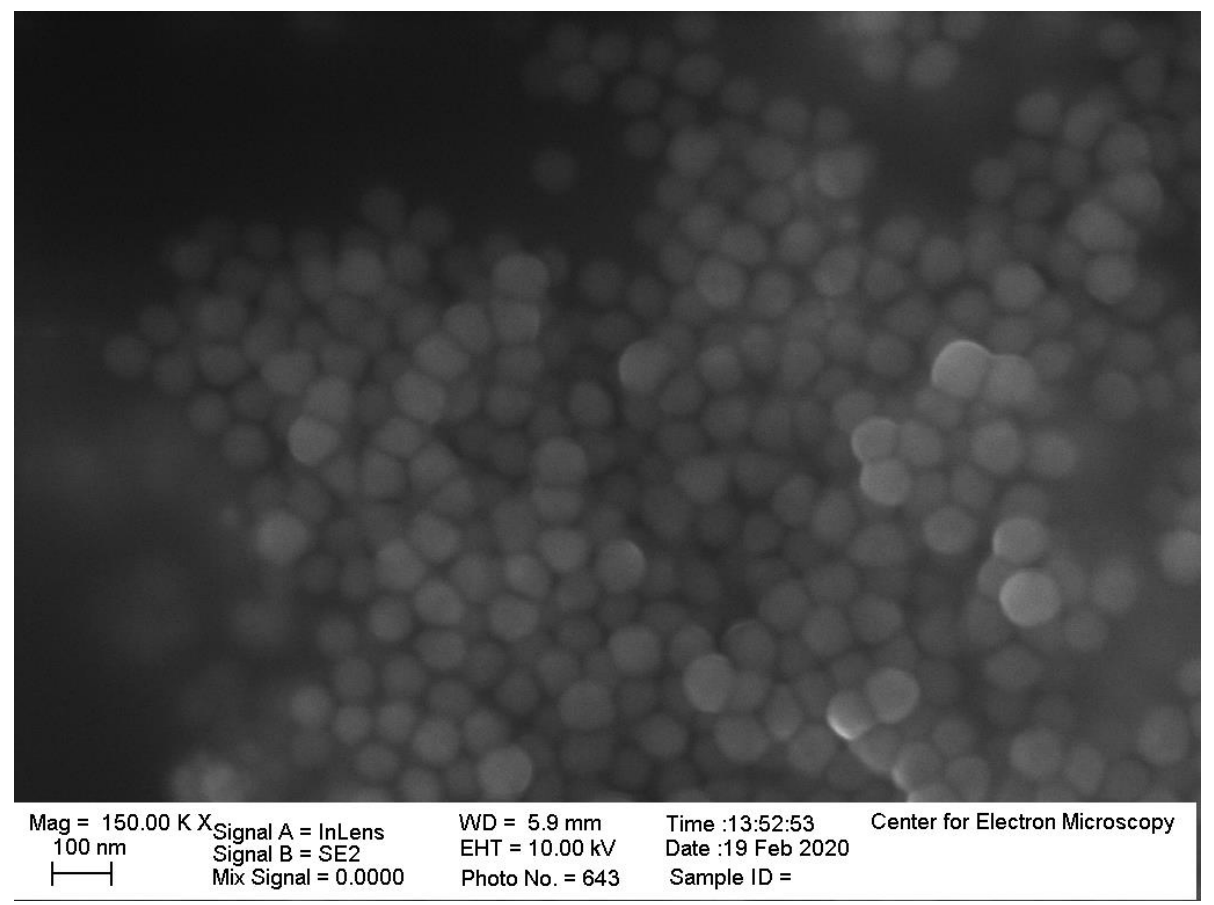

Figure 3.3. High magnification SEM micrograph of NanoXact nanoparticles imaged with the InLens detector, EHT of $10 \mathrm{kV}, 150 \mathrm{kx}$ magnification, and a scale bar of $100 \mathrm{~nm}$.

The morphology of NanoXact particles before magnesiothermic reduction appears to be spherical, and the nanoparticles are in close proximity to one another. Following these images are the NanoXact particles after magnesiothermic reduction with and without $\mathrm{NaCl}$ as a heat sink. 
NanoXact with $\mathrm{NaCl}$ :

Figure 3.4 depicts a low magnification image of the nanoparticles after magnesiothermic reduction with $\mathrm{NaCl}$.

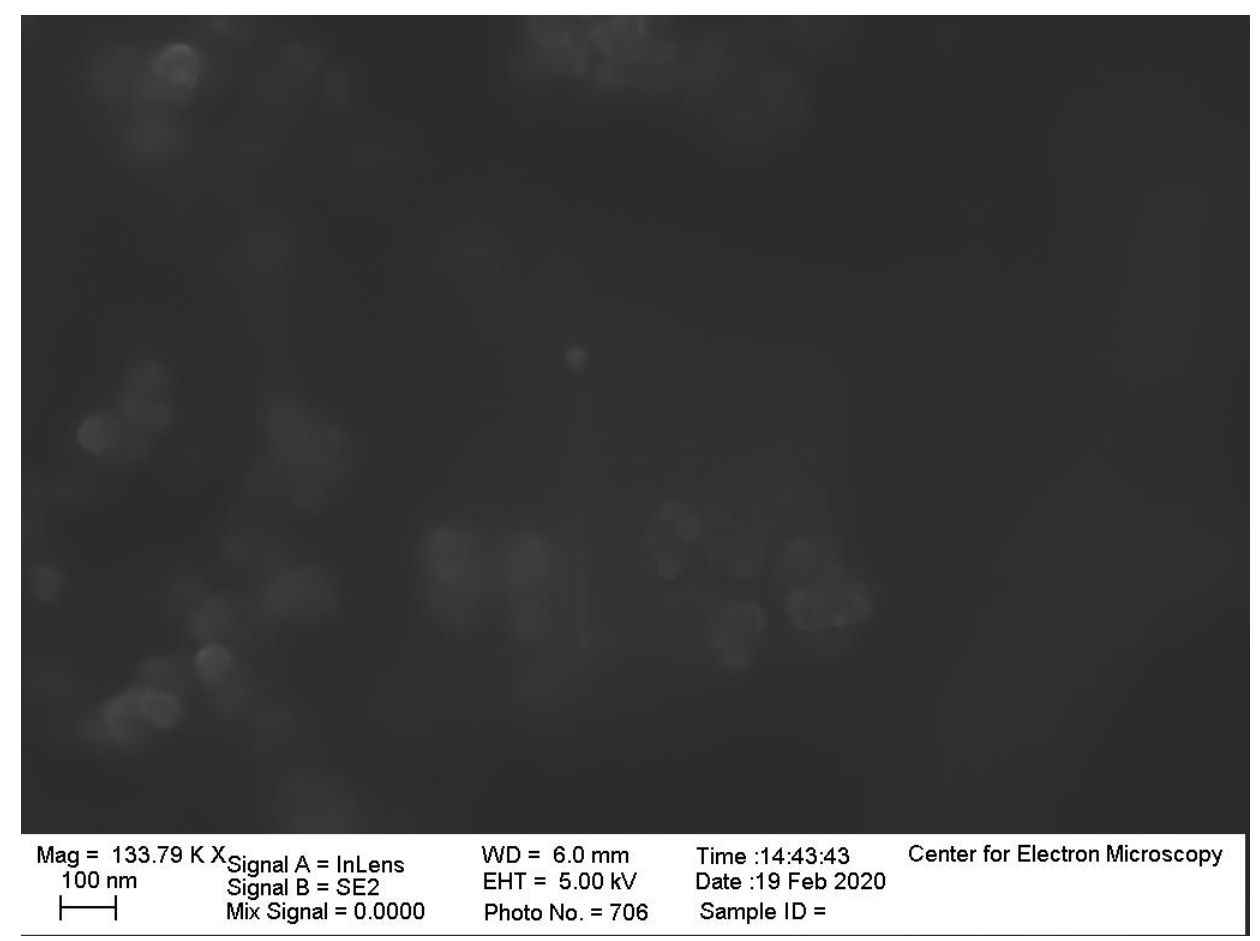

Figure 3.4. High magnification SEM micrograph of NanoXact with $\mathrm{NaCl}$ nanoparticles imaged with the InLens detector, EHT of $5 \mathrm{kV}, 133.79 \mathrm{kx}$ magnification, and a scale bar of $100 \mathrm{~nm}$.

The nanoparticles appear to be spherical but with a nonuniform size distribution. A few nanoparticles have fused together forming irregular non-spherical shapes. Figure 3.5 shows low magnification images of these nanoparticles, which appear to form a large surface containing many nanoparticles with clusters of various heights. 


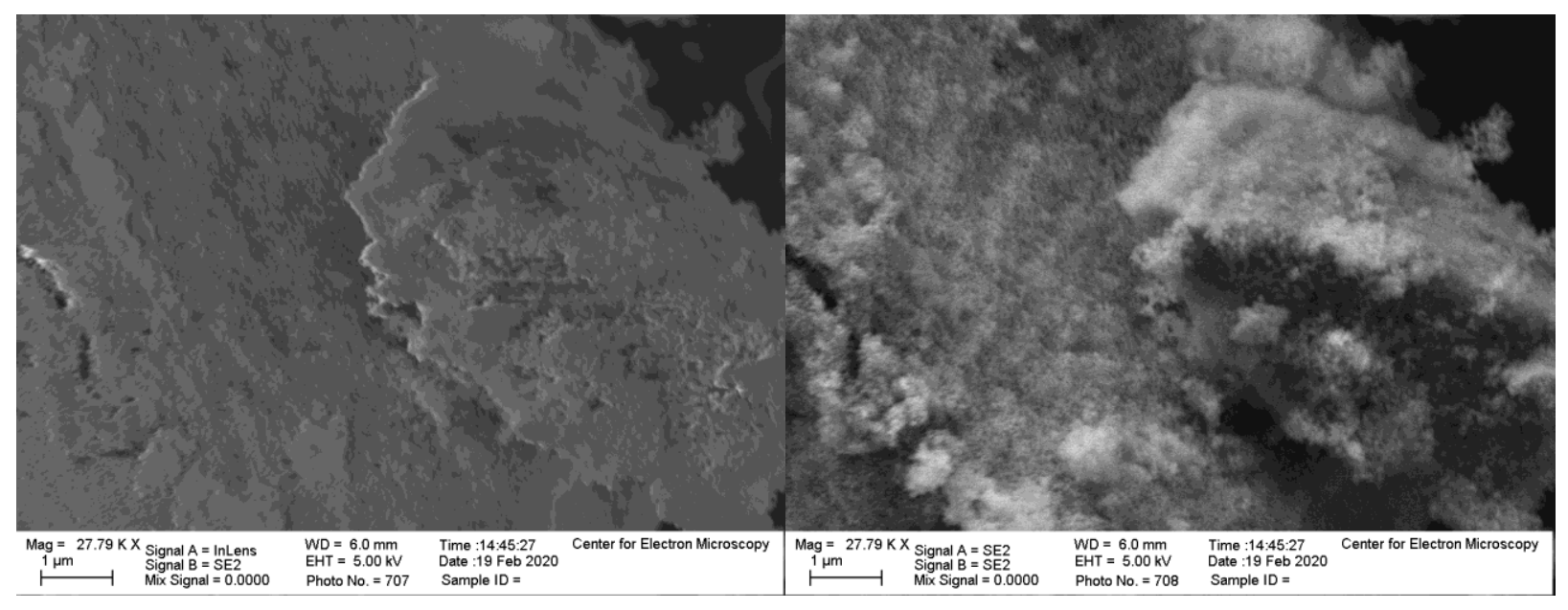

Figure 3.5. Low magnification SEM micrographs of NanoXact with $\mathrm{NaCl}$ nanoparticles, EHT of $5 \mathrm{kV}$, and a $27.79 \mathrm{kx}$ magnification. Left: InLens detector and a scale bar of $1 \mathrm{um}$. Right: SE2 detector and a scale bar of $1 \mathrm{um}$.

Increasing the magnification at this location results in the micrographs displayed in figure 3.6. The image on the bottom has distortion, but both images display spherical particles. It is challenging to determine if the nanospheres have fused or if the topological information is lost from the InLens detector. The secondary detector was unable to image the sample at higher magnification. 


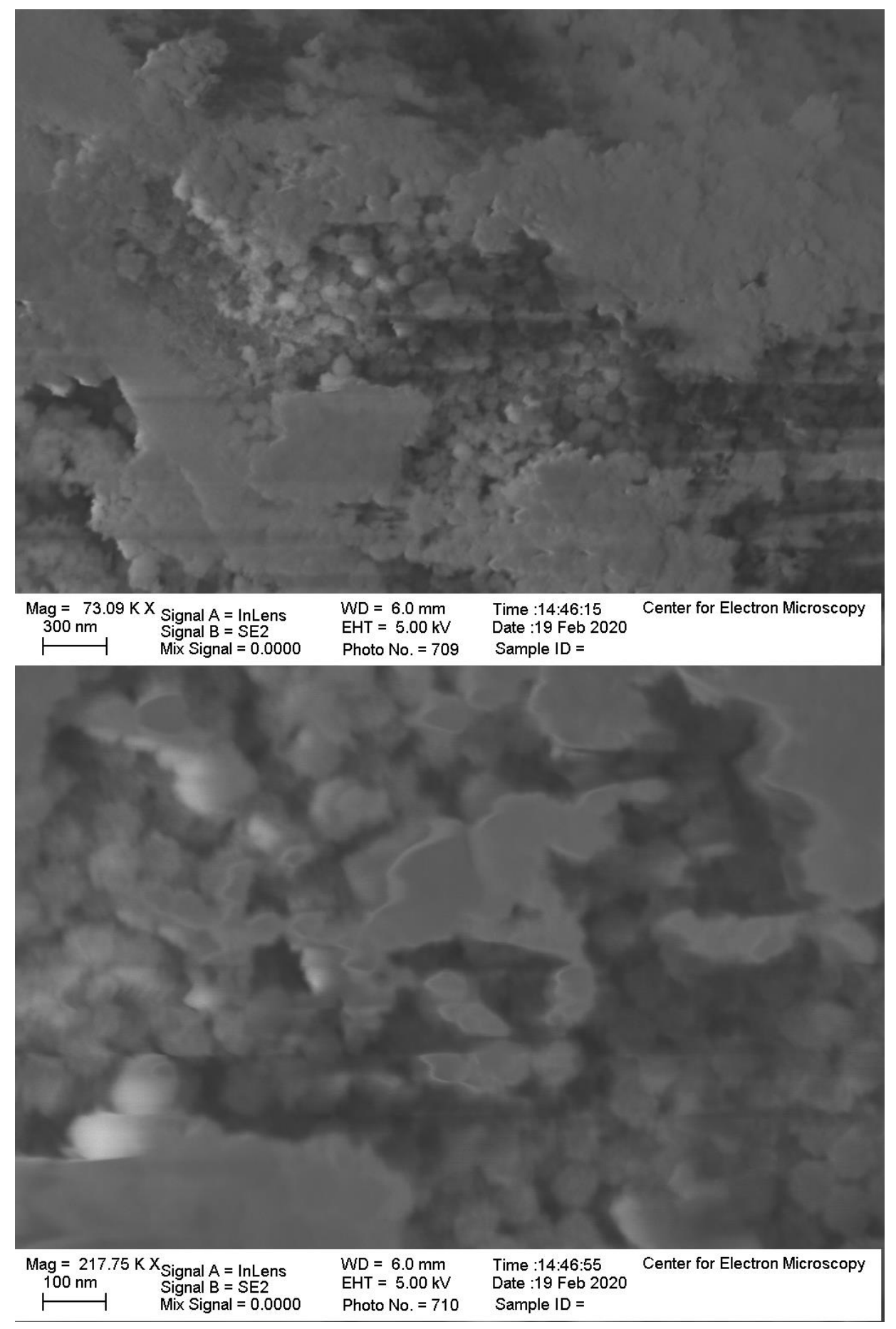

Figure 3.6. SEM micrographs of NanoXact with $\mathrm{NaCl}$ nanoparticles imaged at the same location with EHT of $5 \mathrm{kV}$. Top: lower magnification micrograph, $73.09 \mathrm{kx}$; scale bar of $300 \mathrm{~nm}$.

Bottom: higher magnification micrograph, $217.75 \mathrm{kx}$; scale bar of $100 \mathrm{~nm}$. 


\section{NanoXact without $\mathrm{NaCl}$ :}

At low magnification, the NanoXact nanoparticles following magnesiothermic reduction without

$\mathrm{NaCl}$ as a heat sink look clustered together in clusters of varying sizes.

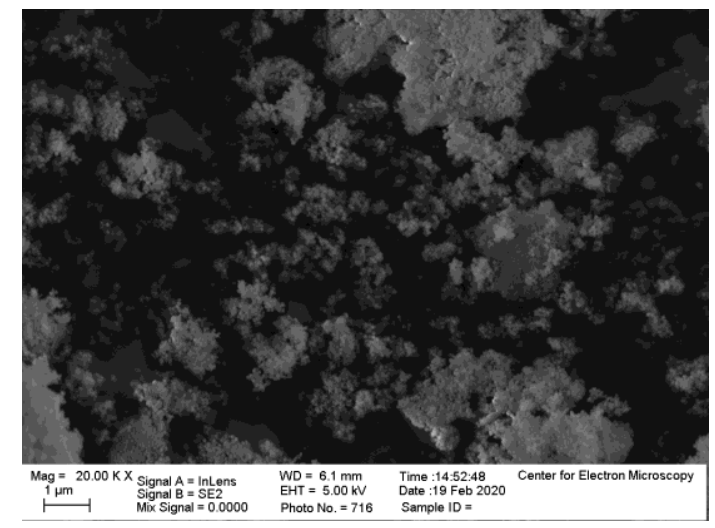

Figure 3.7. Low magnification SEM micrograph of NanoXact nanoparticles without NaCl, EHT of $5 \mathrm{kV}$, InLens detector, a $20 \mathrm{kx}$ magnification, and a $1 \mathrm{um}$ scale bar.

At higher magnification, the NanoXact particles without $\mathrm{NaCl}$ as a heat sink appear to have retained morphology as displayed in Figures 3.8 and 3.9.

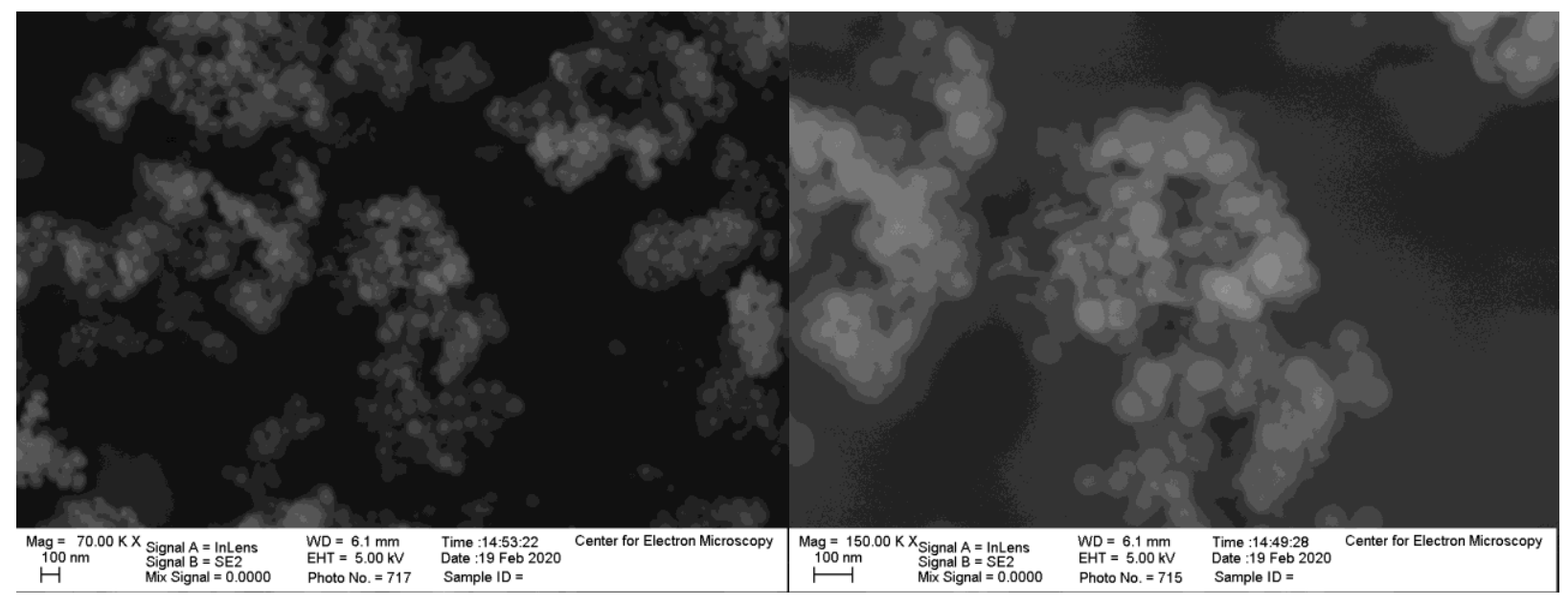

Figure 3.8. SEM micrographs of NanoXact nanoparticles without $\mathrm{NaCl}$, EHT of $5 \mathrm{kV}$, a scale bar of $100 \mathrm{~nm}$, and InLens detector. Left: $70 \mathrm{kx}$ magnification. Right: $150 \mathrm{kx}$ magnification. 


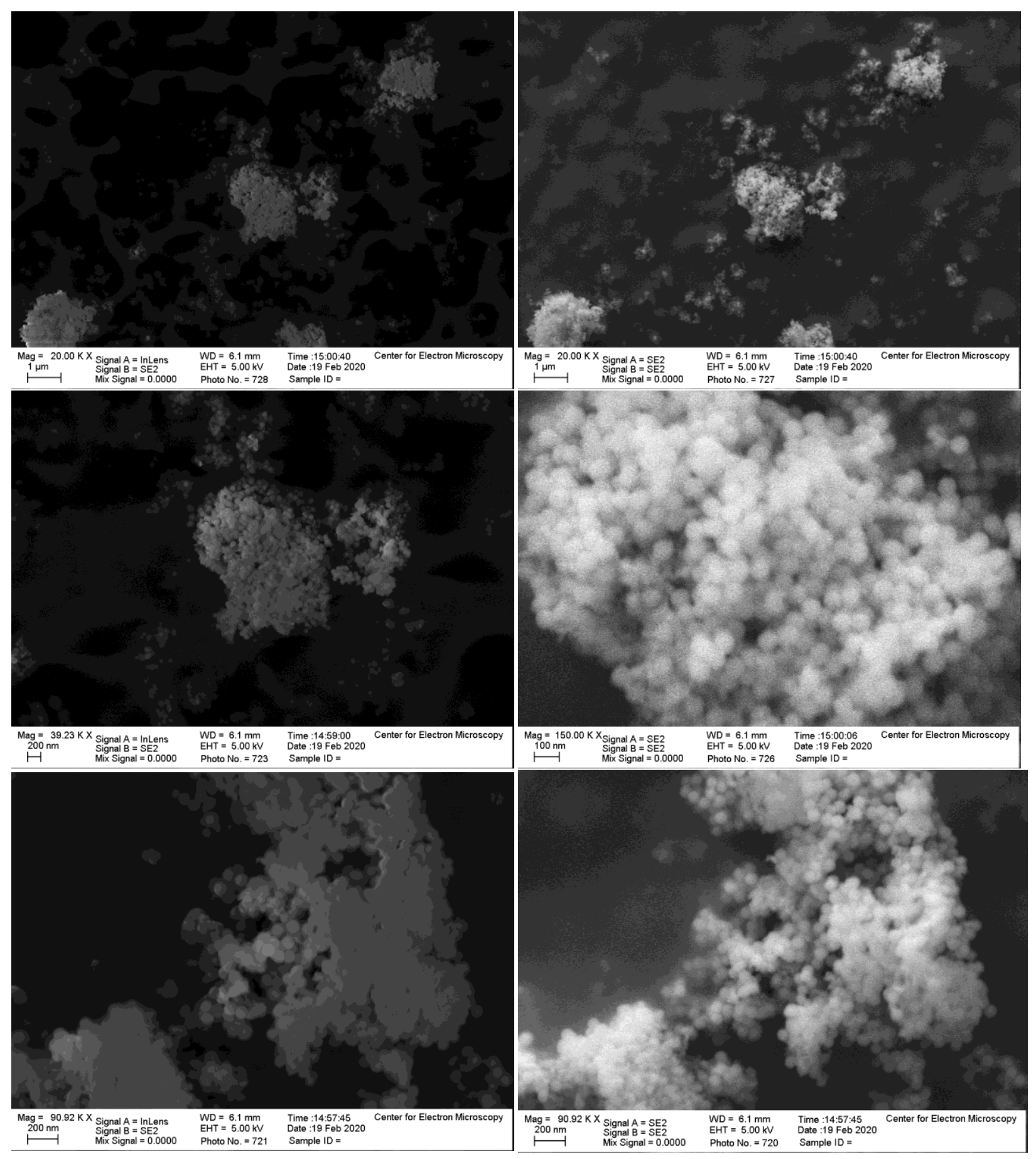

Figure 3.9. Series of SEM micrographs of NanoXact nanoparticles without $\mathrm{NaCl}$. Imaged at the same location with EHT of $5 \mathrm{kV}$, varying magnification, and both detectors. Images on the left are imaged with the InLens detector; images on the right are imaged with the SE2 detector. 

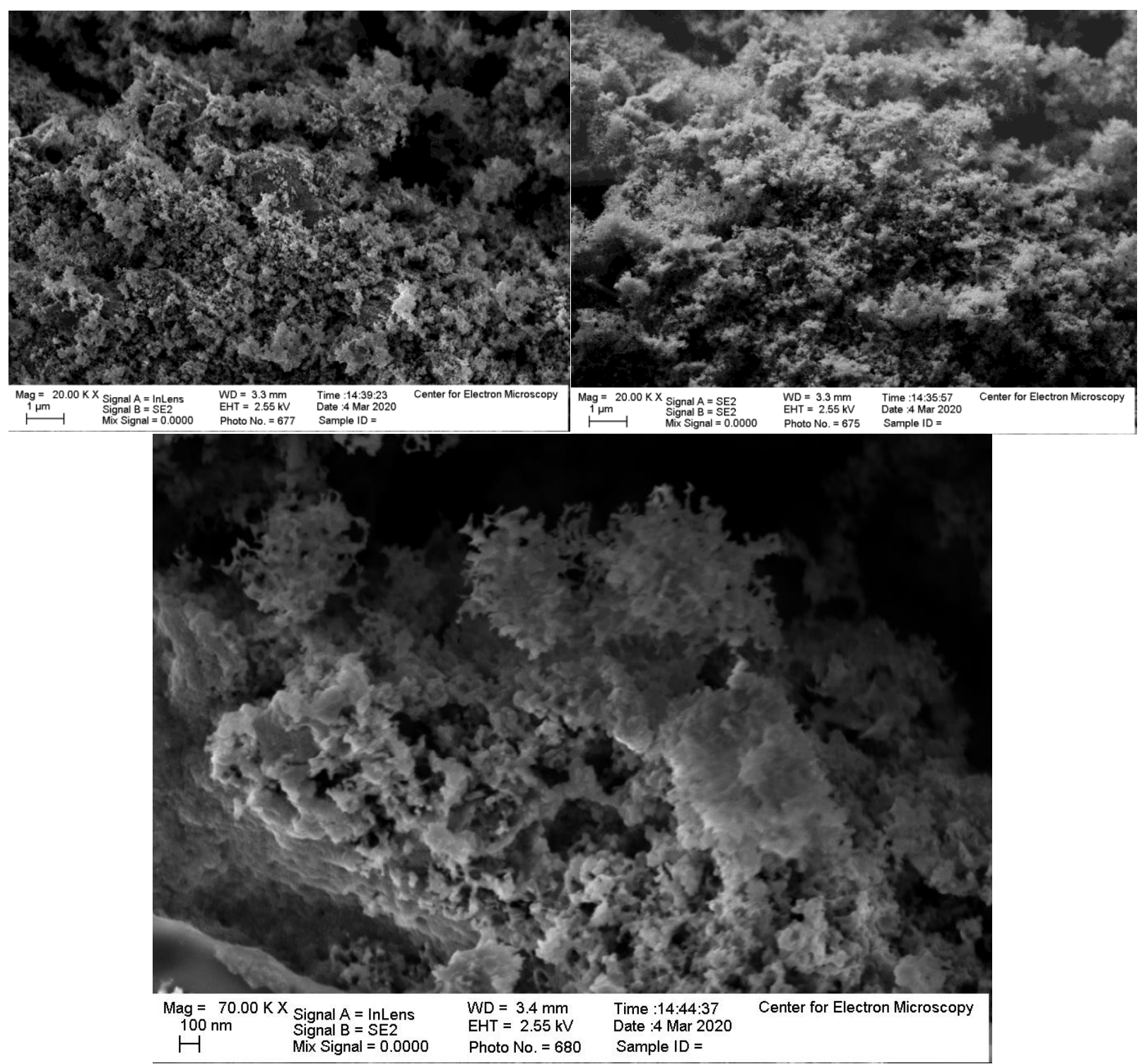

Figure 3.10. Series of SEM micrographs of NanoXact nanoparticles with $\mathrm{NaCl}$ after the $\mathrm{HF}$ etching process imaged at EHT of $2.55 \mathrm{kV}$. Top micrographs were imaged at the same location with $20 \mathrm{kx}$ magnification and a scale bar of $1 \mathrm{um}$. The top left was imaged with the InLens detector, and the right was imaged with the SE2 detector. The bottom micrograph was imaged at $70 \mathrm{kx}$ and a scale bar of $100 \mathrm{~nm}$ with the InLens detector. 
After magnesiothermic reduction with $\mathrm{NaCl}$, the NanoXact particles were etched with $\mathrm{HF}$ to eliminate any silica byproduct. The low magnification images in Figure 3.10 display varying sizes of silicon clusters. We were unable to image higher magnifications of the NanoXact nanoparticles with $\mathrm{NaCl}$ after the $\mathrm{HF}$ etch due to image distortion, but at $70 \mathrm{kx}$ magnification, the particles appear to be fused, forming amorphous shapes.

NanoXact without $\mathrm{NaCl}$ after HF etch:

Similarly to the NanoXact nanoparticles with $\mathrm{NaCl}$ after the $\mathrm{HF}$ wash, the NanoXact nanoparticles without $\mathrm{NaCl}$ after the $\mathrm{HF}$ etch appear to be amorphous, as depicted in Figures 3.11 and 3.12. 


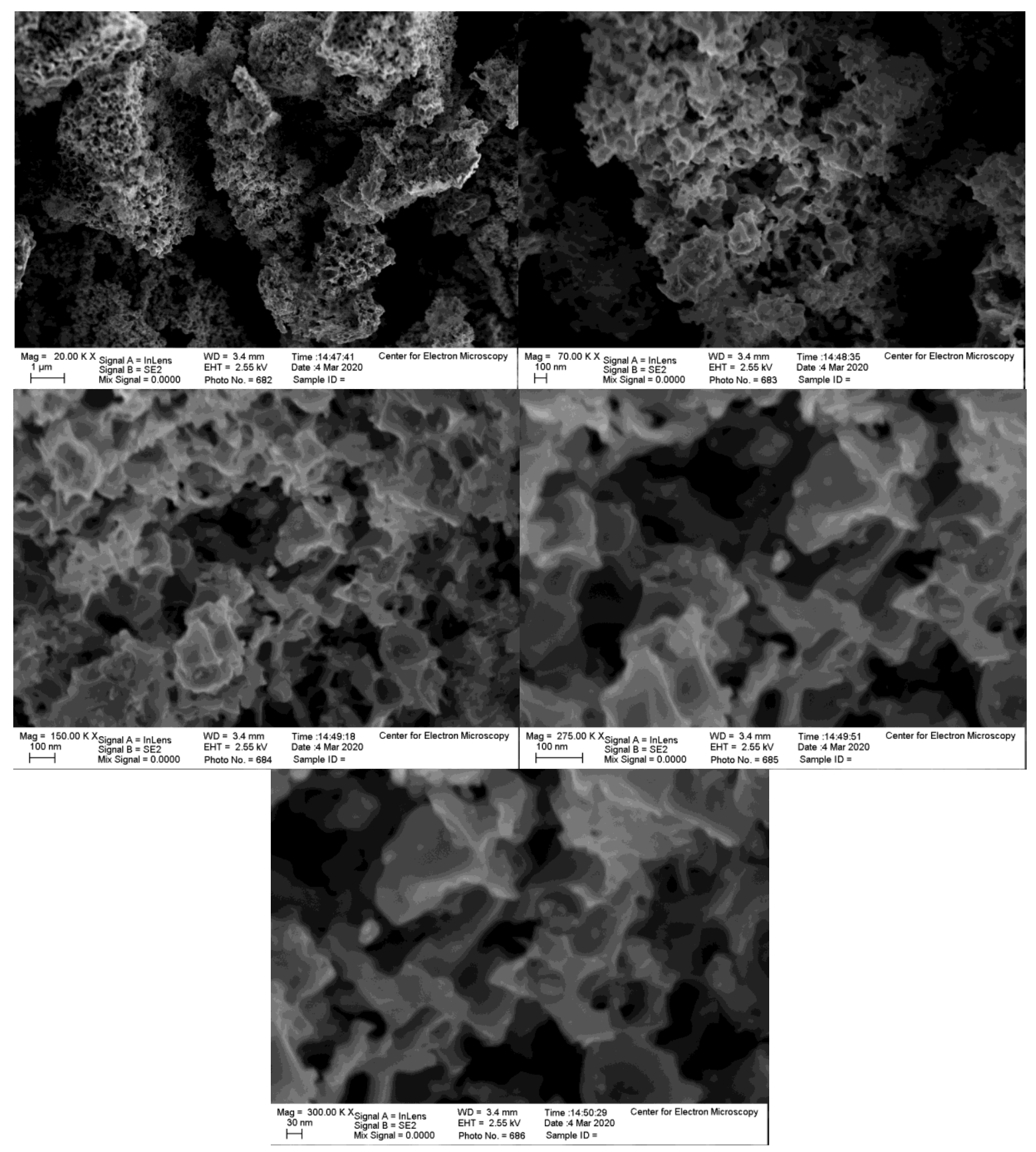

Figure 3.11. Series of SEM micrographs of NanoXact nanoparticles without $\mathrm{NaCl}$ after the $\mathrm{HF}$ etching process imaged at EHT of $2.55 \mathrm{kV}$ with the InLens detector. The top left was imaged with $20 \mathrm{kx}$, and a scale bar of $1 \mathrm{um}$. The top right was imaged at $70 \mathrm{kx}$ with a $100 \mathrm{~nm}$ scale bar. The following images were imaged at $150 \mathrm{kx}, 275 \mathrm{kx}$, and $300 \mathrm{kx}$ with scale bars of $100 \mathrm{~nm}, 100$ $\mathrm{nm}$, and $30 \mathrm{~nm}$ respectively. 


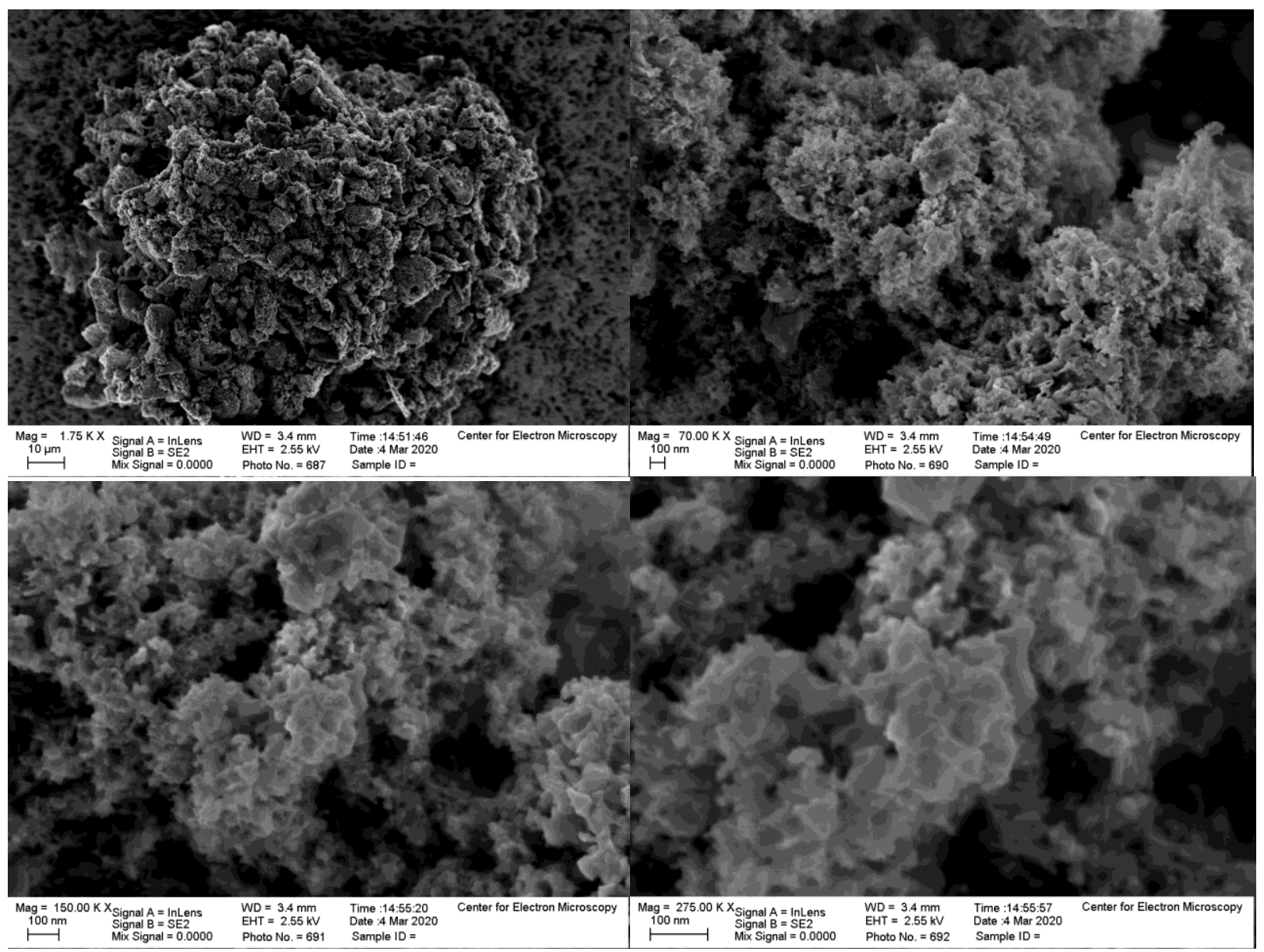

Figure 3.12. Series of SEM micrographs of NanoXact nanoparticles with $\mathrm{NaCl}$ after the $\mathrm{HF}$ etching process imaged at EHT of $2.55 \mathrm{kV}$ with the InLens detector. The top left micrograph was imaged at $1.75 \mathrm{kx}$, and a scale bar of $10 \mathrm{um}$. The following images have a scale bar of $100 \mathrm{~nm}$. The top right was imaged with $70 \mathrm{kx}$ magnification. The bottom left micrograph was imaged at $150 \mathrm{kx}$, and the bottom right was imaged at $275 \mathrm{kx}$ magnification. 
Stöber:

The Stöber nanoparticles prior to magnesiothermic reduction are solid and spherical, as depicted in Figures 3.13 and 3.14.

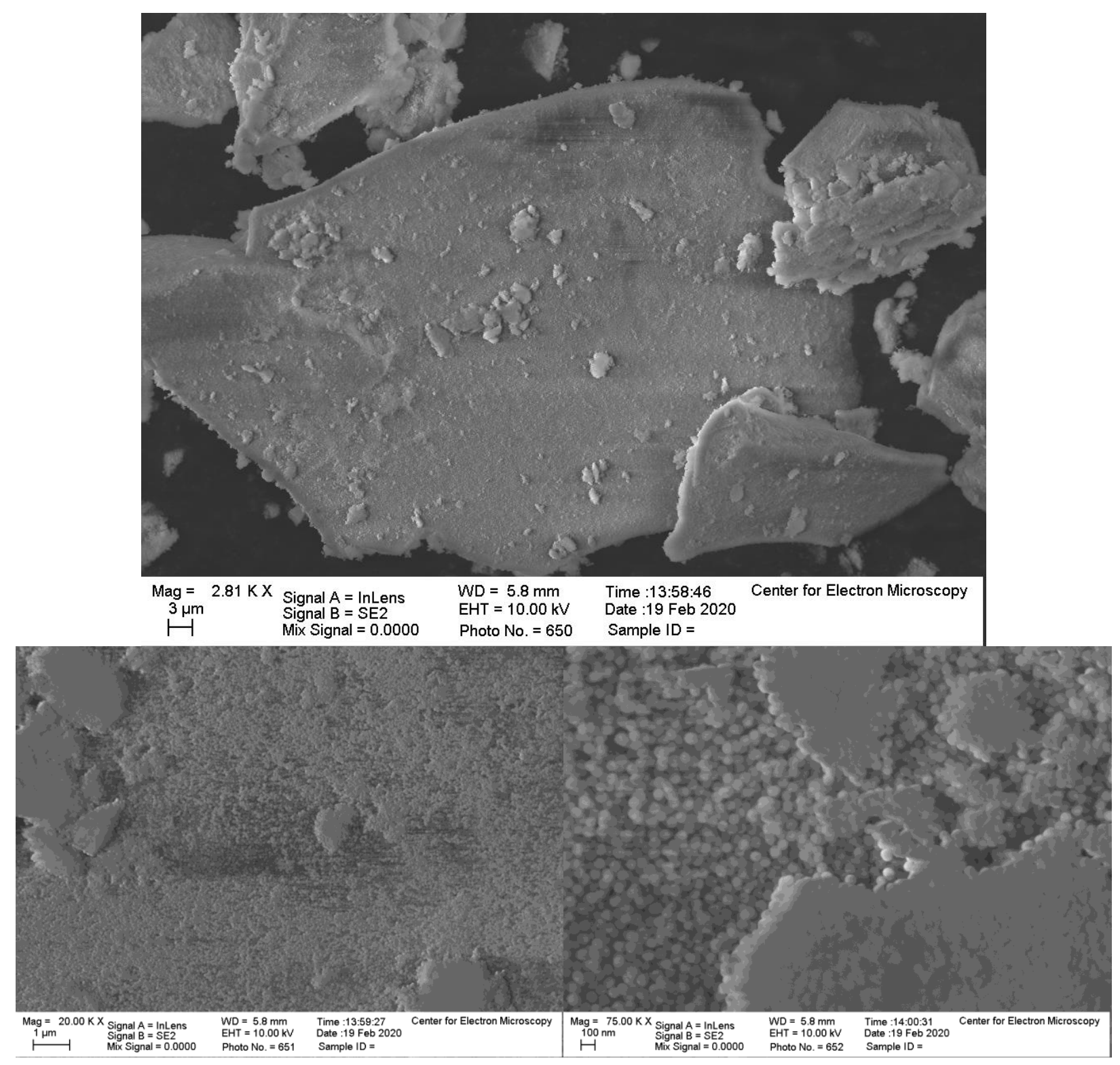

Figure 3.13. Series of SEM micrographs of Stöber nanoparticles imaged at EHT of $10 \mathrm{kV}$ with the InLens detector. The top micrograph was imaged at $2.81 \mathrm{kx}$ and a scale bar of $3 \mathrm{um}$. The 
bottom left micrograph was imaged at $20 \mathrm{kx}$ and a scale bar of $1 \mathrm{um}$. The bottom right was imaged at $75 \mathrm{kx}$ magnification and a scale bar of $100 \mathrm{~nm}$.

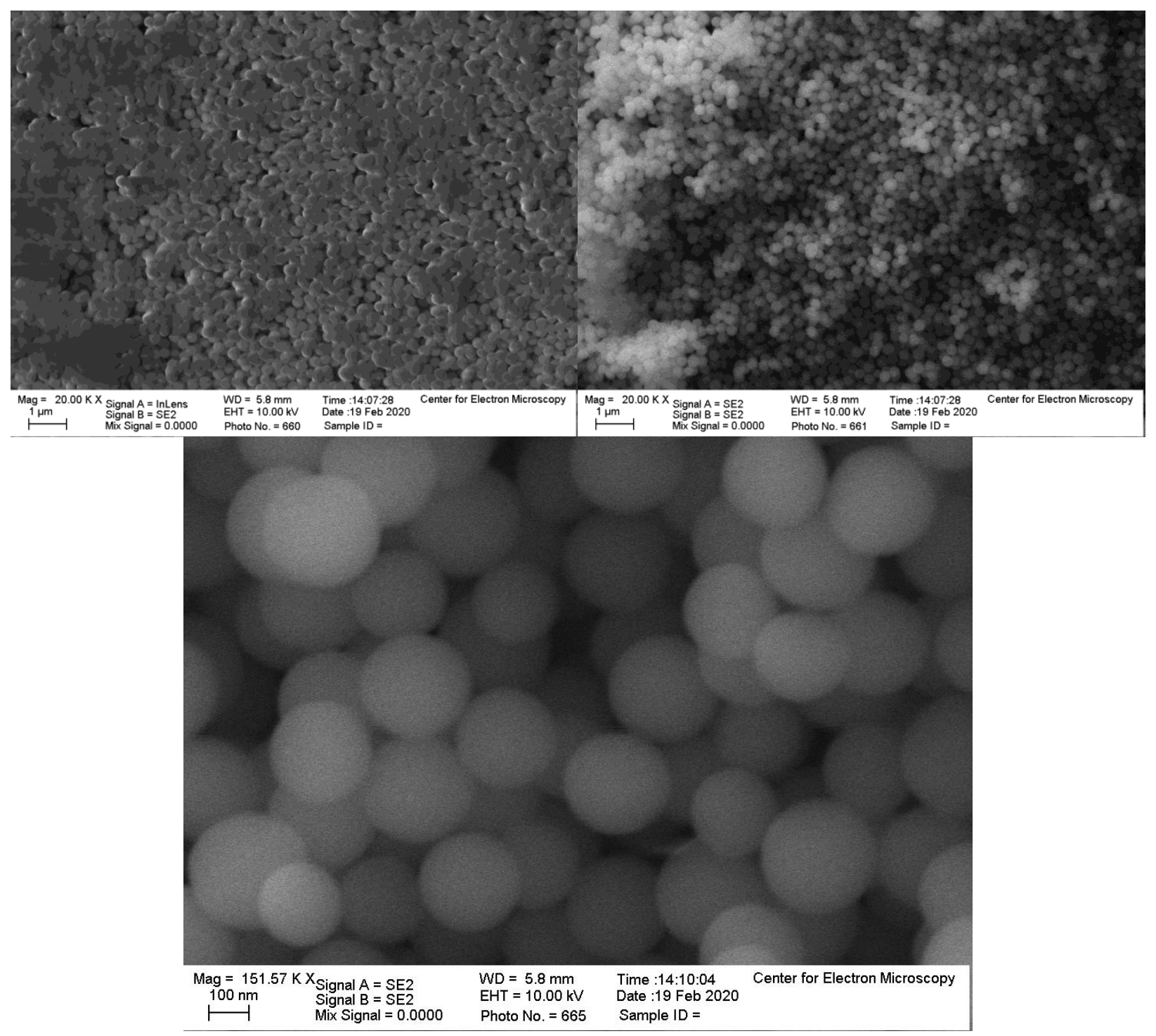

Figure 3.14. Series of SEM micrographs of Stöber nanoparticles imaged at EHT of $10 \mathrm{kV}$. The top micrographs were imaged at the same location, $20 \mathrm{kx}$ magnification, and a scale bar of $1 \mathrm{um}$. The top left was imaged with the InLens detector, and the top right was imaged with the SE2 detector. The bottom micrograph was imaged at $151.57 \mathrm{kx}$ and a scale bar of $100 \mathrm{~nm}$ with the SE2 detector. 
Stöber with $\mathrm{NaCl}$ :

Following magnesiothermic reduction with $\mathrm{NaCl}$ as a heat sink, the Stöber nanoparticles retained morphology in comparison to the starting material. However, there are impurities or fusion of particles, as seen in Figures 3.15 and 3.16.

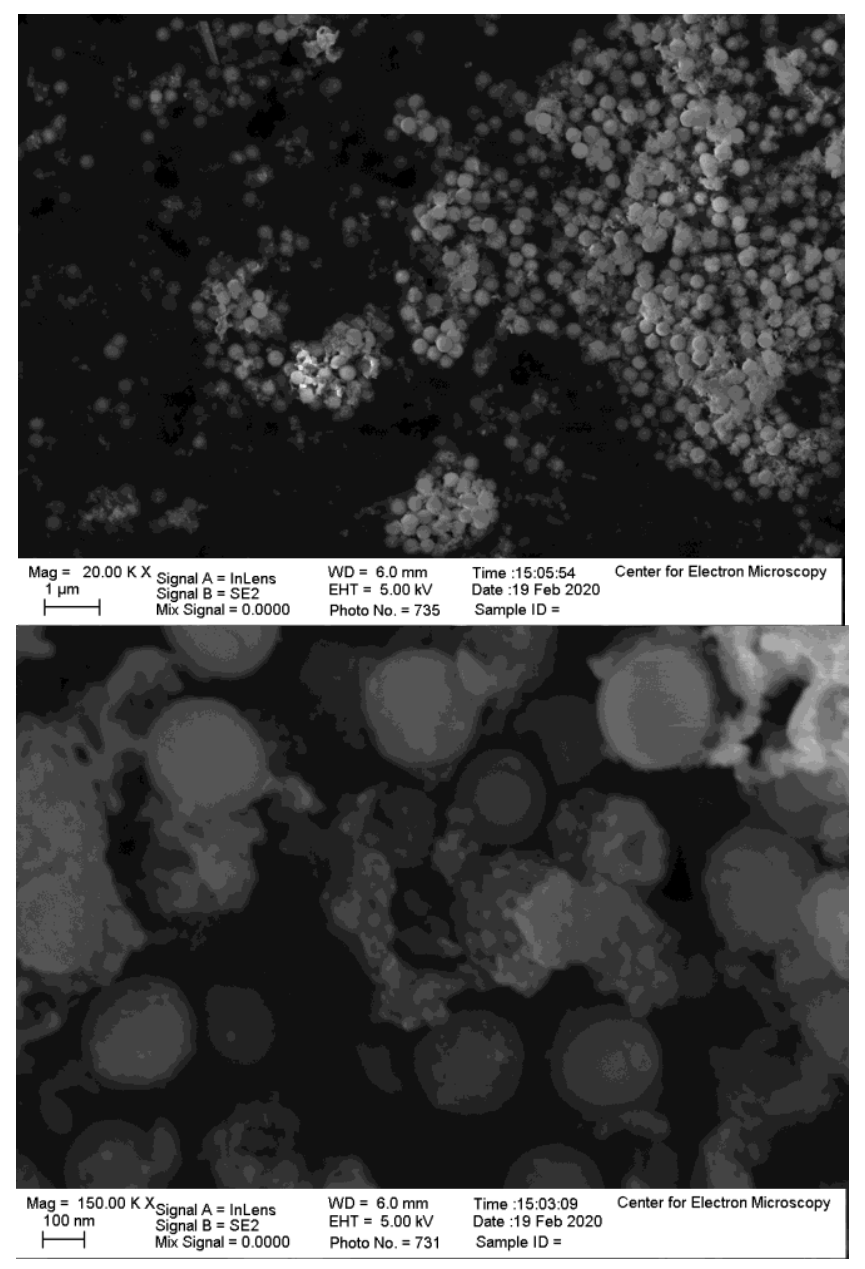

Figure 3.15. SEM micrographs of Stöber nanoparticles with $\mathrm{NaCl}$ imaged at EHT of $5 \mathrm{kV}$ with the InLens detector. The top micrograph was imaged at $20 \mathrm{kx}$ and has a scale bar of $1 \mathrm{um}$. The bottom micrograph was imaged at $150 \mathrm{kx}$ and has a scale bar of $100 \mathrm{~nm}$. 


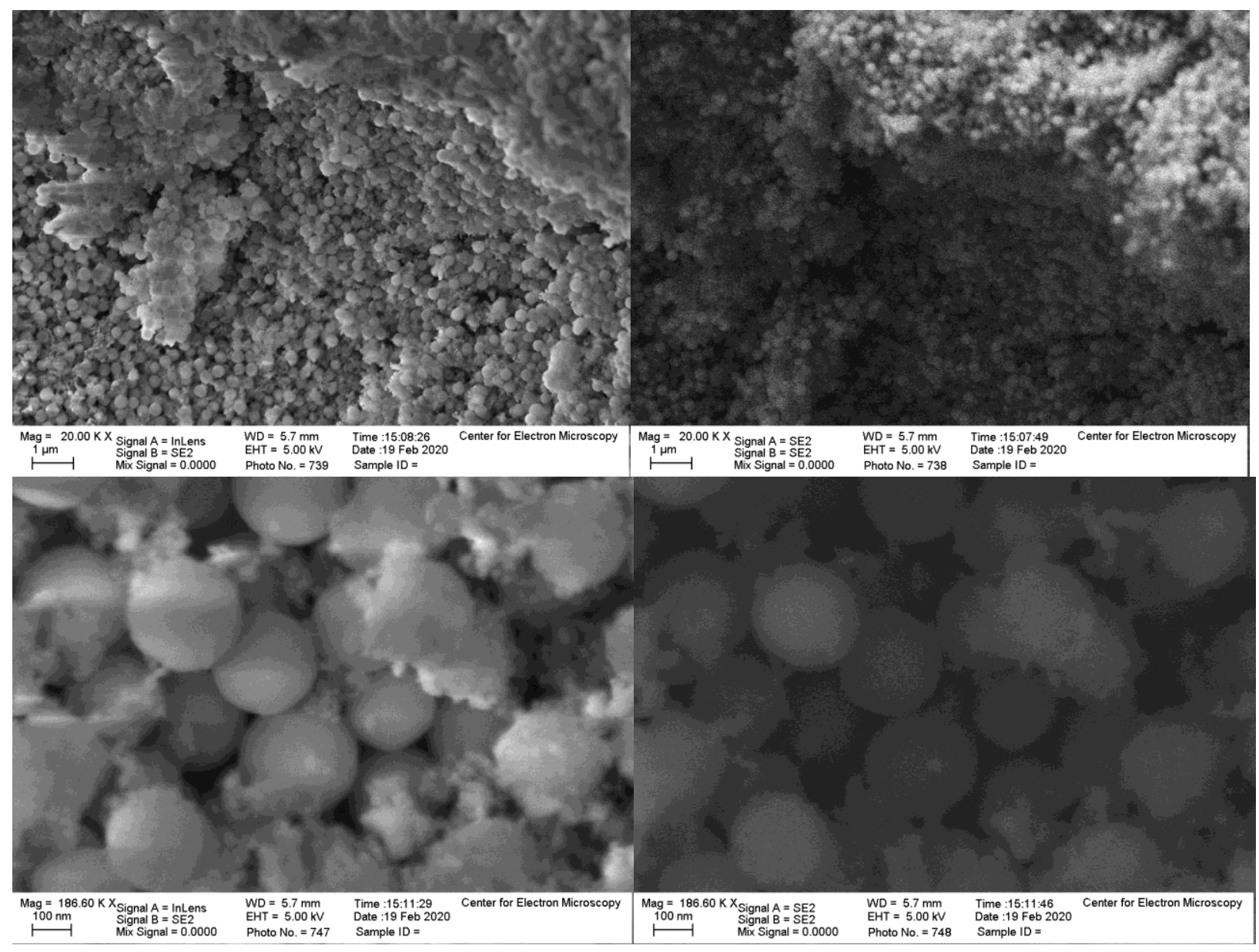

Figure 3.16. SEM micrographs of Stöber nanoparticles with $\mathrm{NaCl}$ imaged at EHT of $5 \mathrm{kV}$. The top micrographs were imaged at the same location, have a scale bar of $1 \mathrm{um}$, and $20 \mathrm{kx}$ magnification. The bottom micrographs were imaged at the same location, with $186.60 \mathrm{kx}$ and a scale bar of $100 \mathrm{~nm}$. Images on the left: InLens, images on the right: SE2. 
Stöber without $\mathrm{NaCl}$ :

Stöber nanoparticles that did not have $\mathrm{NaCl}$ as a heat sink fused together and became amorphous as displayed in Figures 3.17, 3.18, and 3.19.

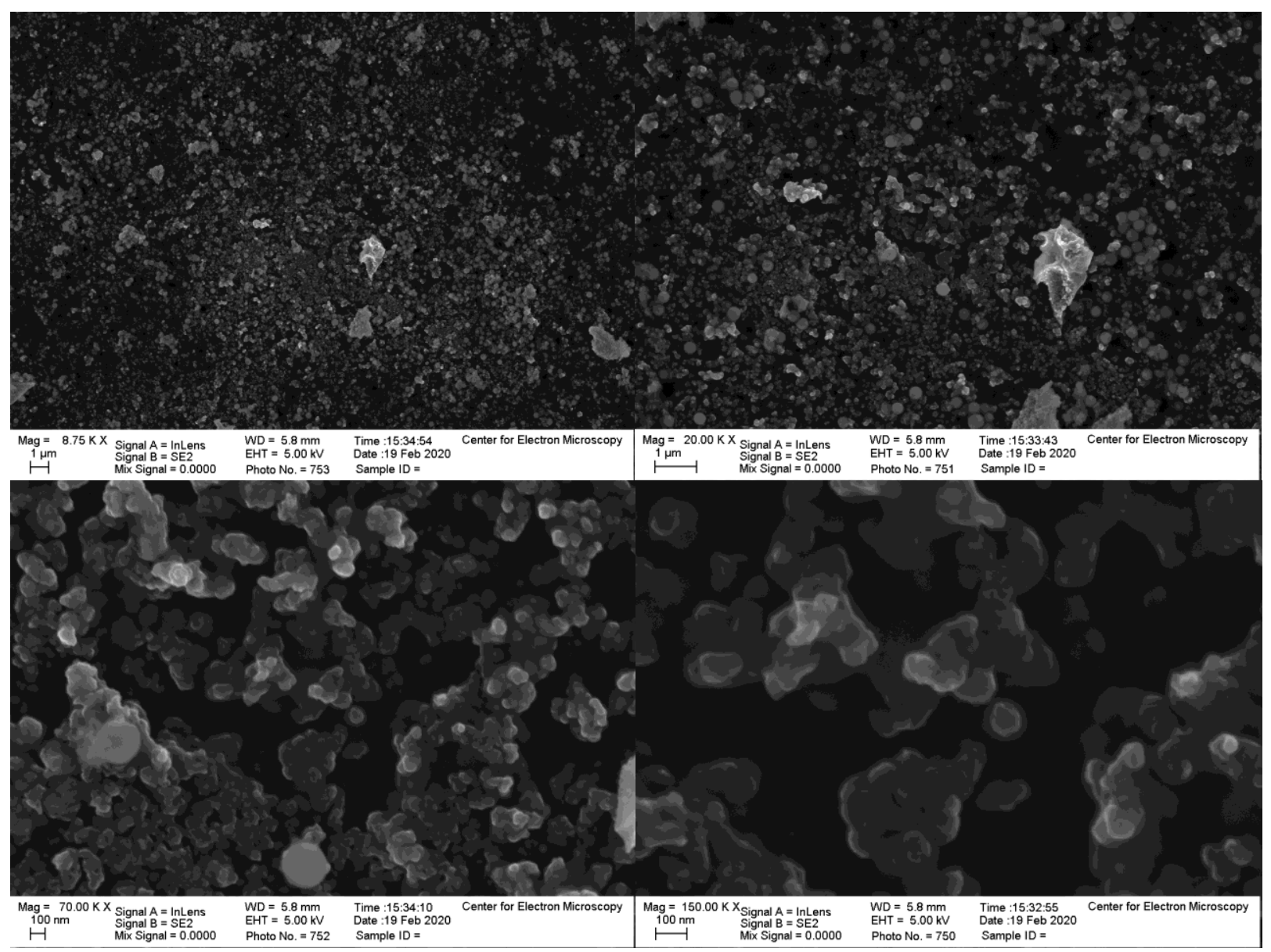

Figure 3.17. SEM micrographs of Stöber nanoparticles without $\mathrm{NaCl}$ imaged at EHT of $5 \mathrm{kV}$ and the InLens detector at the same location with varying magnification. The magnification is $8.75 \mathrm{kx}, 20 \mathrm{kx}, 70 \mathrm{kx}$, and $150 \mathrm{kx}$ with a scale bar of $1 \mathrm{um}, 1 \mathrm{um}, 100 \mathrm{~nm}$, and 100nm, respectively. 


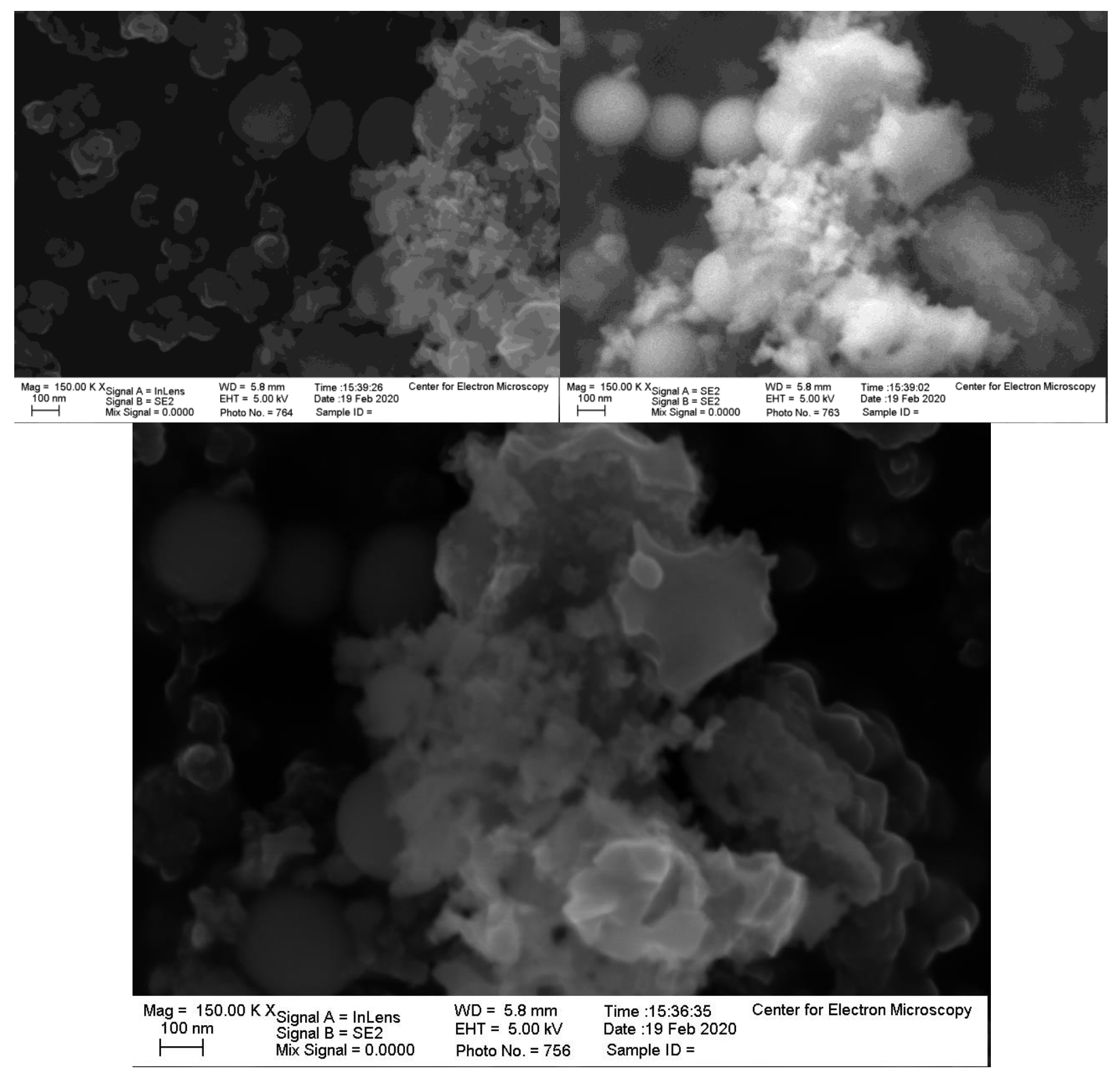

Figure 3.18. SEM micrographs of Stöber nanoparticles without $\mathrm{NaCl}$ imaged at EHT of $5 \mathrm{kV}$ and the InLens detector at the same location, scale bar of $100 \mathrm{~nm}$, and $150 \mathrm{kx}$ magnification. 


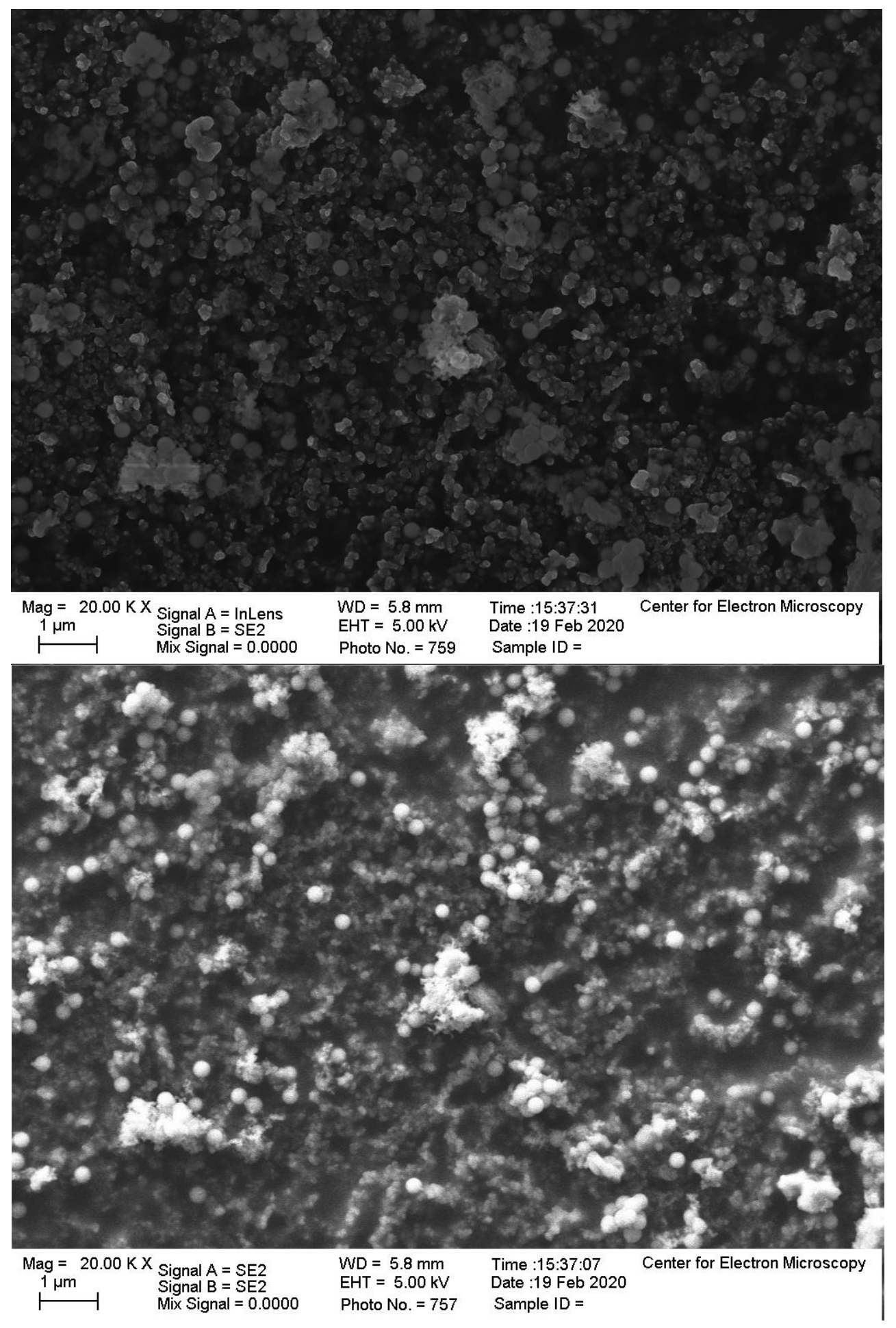

Figure 3.19. SEM micrographs of Stöber nanoparticles without $\mathrm{NaCl}$ imaged at EHT of $5 \mathrm{kV}$ at the same location with $20 \mathrm{kx}$ magnification and a scale bar of $1 \mathrm{um}$. Top: InLens detector. Bottom: SE2 detector. 
Stöber with $\mathrm{NaCl}$ after $\mathrm{HF}$ etch:

Following Magnesiothermic reduction with $\mathrm{NaCl}$ as a heat sink and the $\mathrm{HF}$ etching process, these Stöber nanoparticles fused together to form an amorphous shape, as seen in Figures 3.20, 3.21, 3.22, and 3.23.

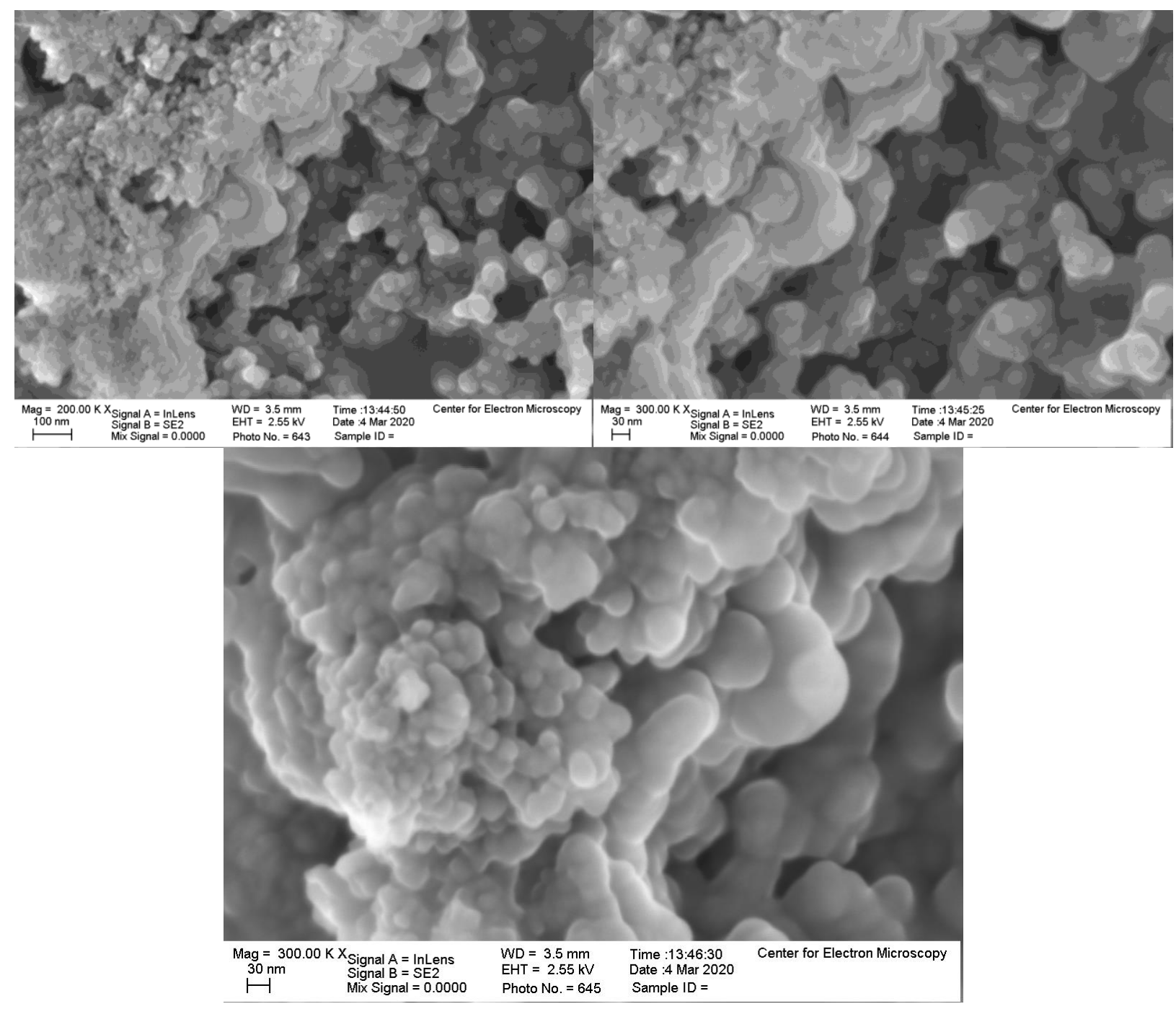

Figure 3.20. SEM micrographs of Stöber nanoparticles with $\mathrm{NaCl}$ after being etched with $\mathrm{HF}$ imaged at EHT of $2.55 \mathrm{kV}$ at the same location. From top left to right, the magnification was 200 $\mathrm{kx}, 300 \mathrm{kx}$, and $300 \mathrm{kx}$; the scale bars are $100 \mathrm{~nm}, 30 \mathrm{~nm}$, and $30 \mathrm{~nm}$. Left: InLens detector. Right: SE2 detector. 


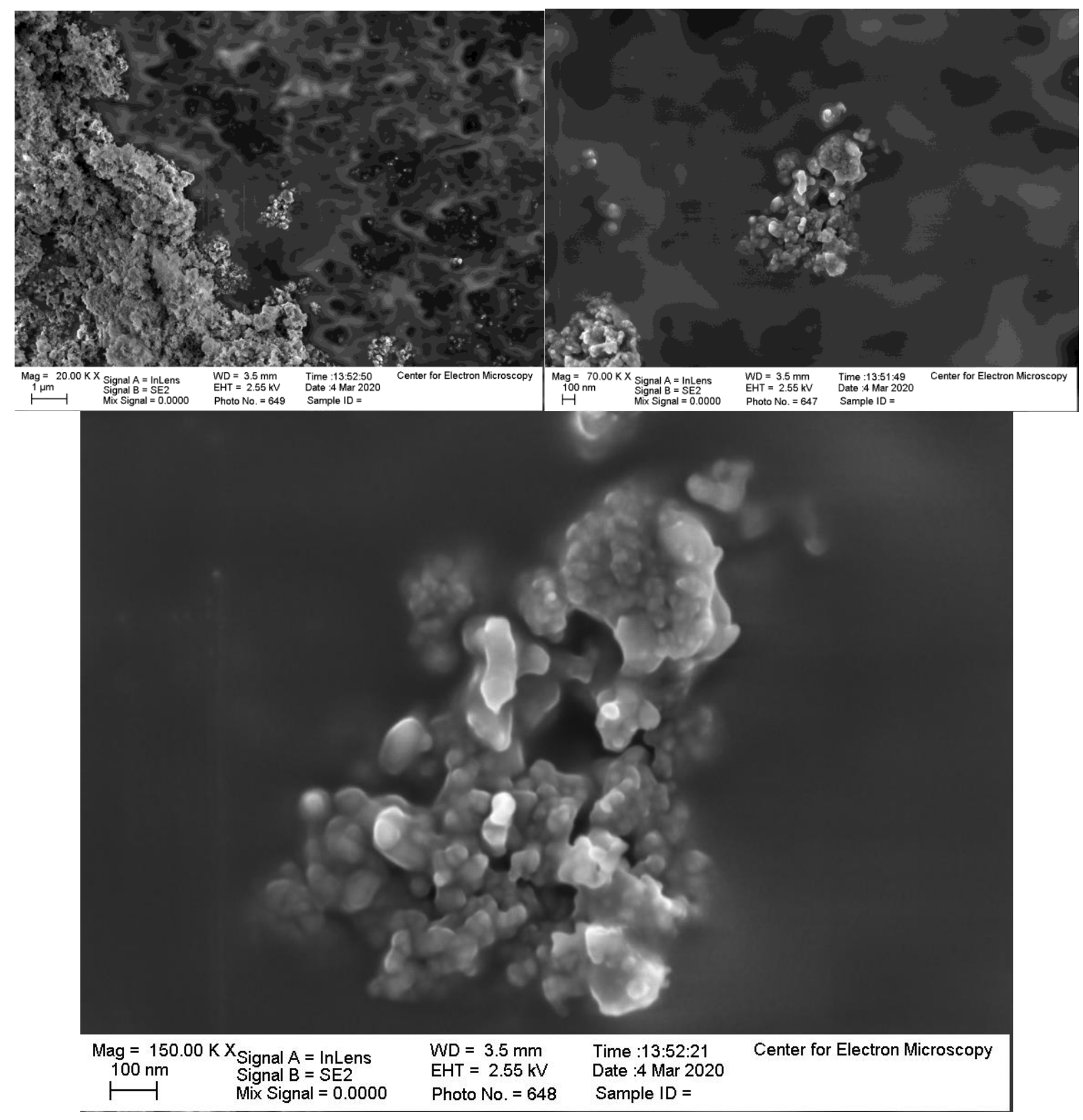

Figure 3.21. SEM micrographs of Stöber nanoparticles with $\mathrm{NaCl}$ after being etched with $\mathrm{HF}$ imaged at EHT of $2.55 \mathrm{kV}$ at the same location with the InLens detector. Top left: $20 \mathrm{kx}$ with a scale bar of $1 \mathrm{um}$. Top right: $70 \mathrm{kx}$, scale bar of $100 \mathrm{~nm}$. Bottom: $150 \mathrm{kx}$, scale bar of $100 \mathrm{~nm}$. 


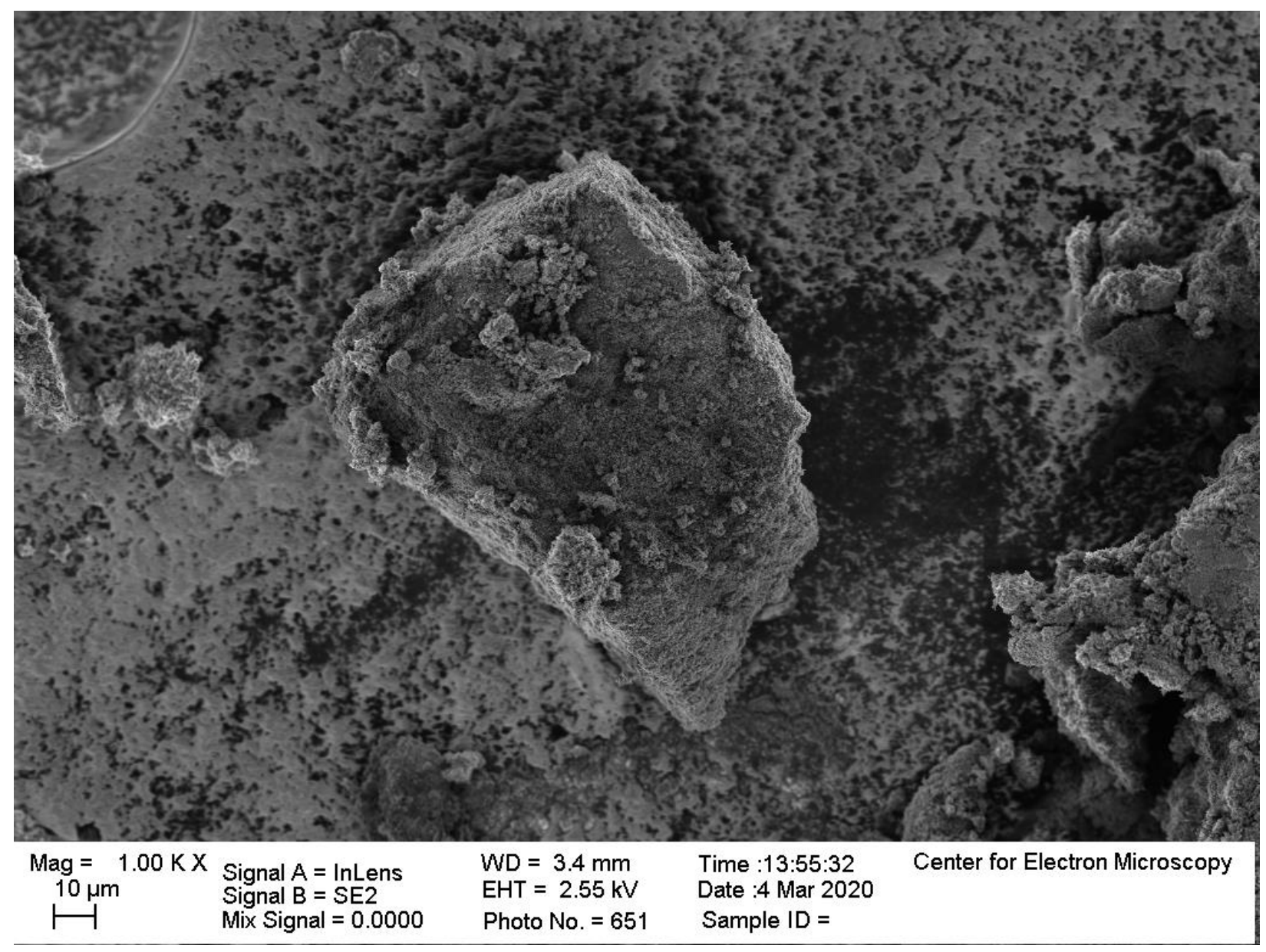

Figure 3.22. SEM micrograph of Stöber nanoparticles with $\mathrm{NaCl}$ after being etched with $\mathrm{HF}$ imaged at EHT of $2.55 \mathrm{kV}$ with the InLens detector, a magnification of $1.00 \mathrm{kx}$, and scale bar of $10 \mathrm{um}$. 


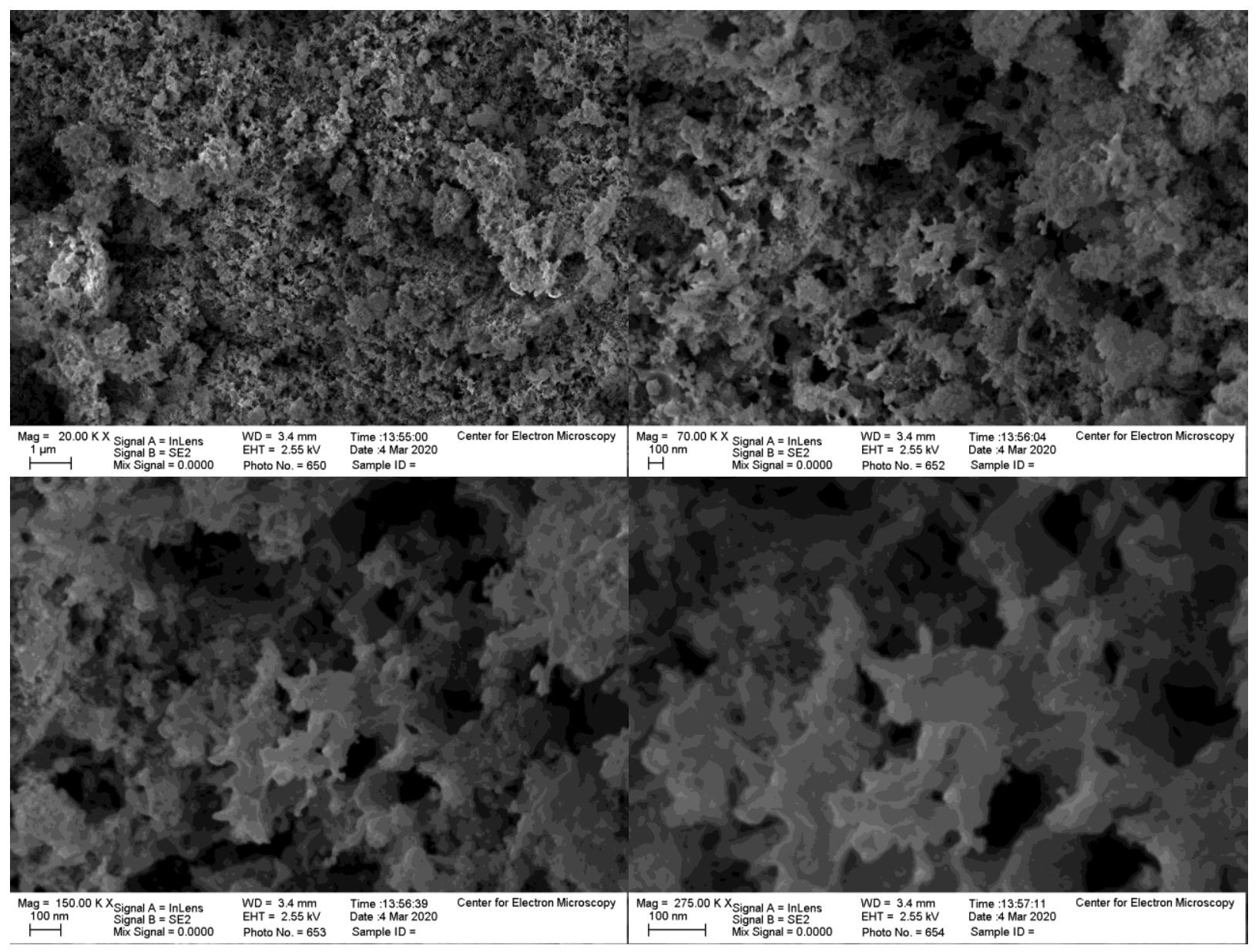

Figure 3.23. SEM micrograph of Stöber nanoparticles with $\mathrm{NaCl}$ after being etched with $\mathrm{HF}$ imaged at EHT of $2.55 \mathrm{kV}$ with the InLens detector. Top left: $20 \mathrm{kx}$ magnification and a scale bar of $1 \mathrm{um}$. The following images have a scale bar of $100 \mathrm{~nm}$. Top right: $70 \mathrm{kx}$ magnification. Bottom left: $150 \mathrm{kx}$ magnification. Bottom right: $275 \mathrm{kx}$ magnification.

Some nanoparticles isolated from the cluster appear to have retained spherical morphology, as illustrated in Figure 3.24. 


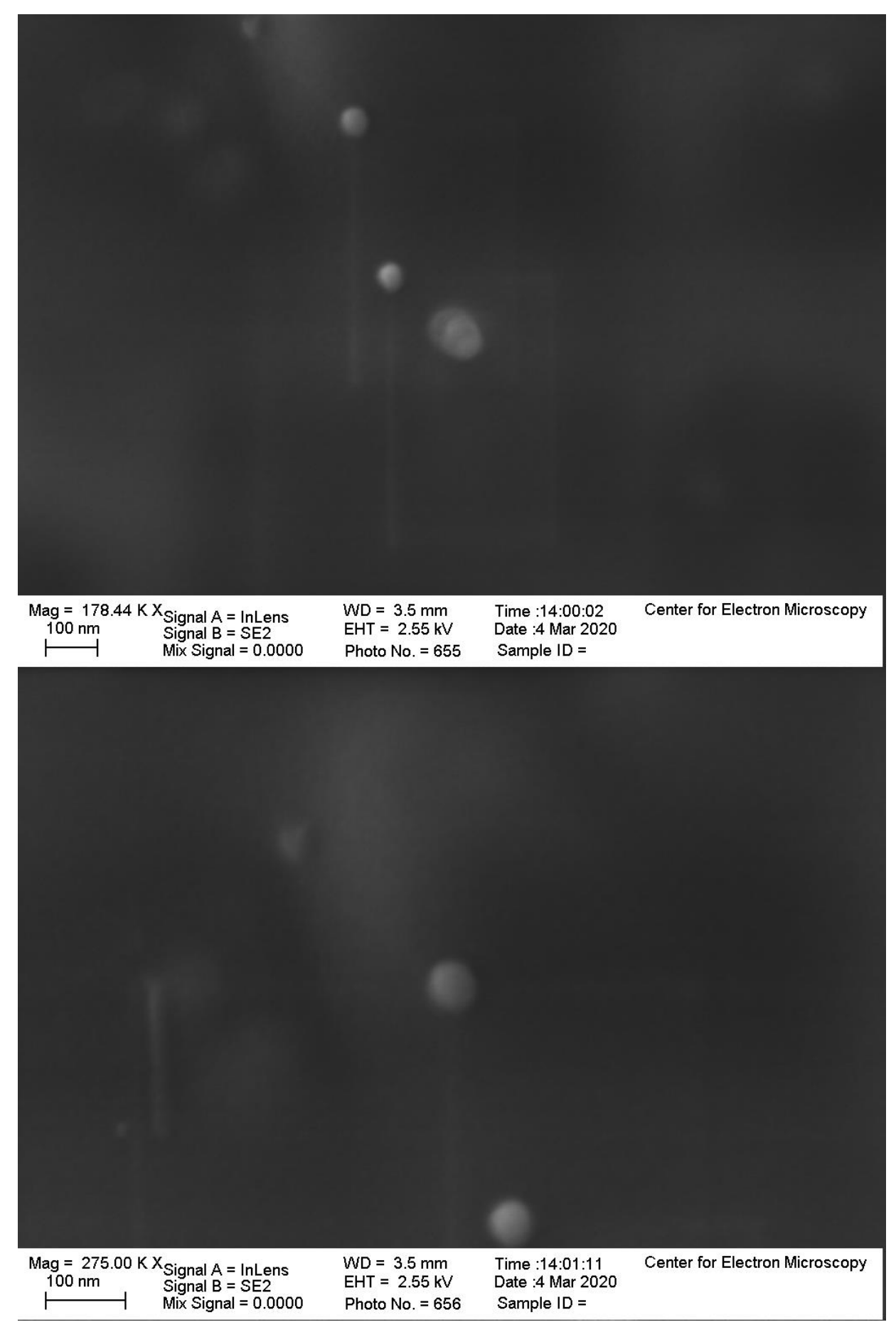

Figure 3.24. SEM micrograph of Stöber nanoparticles with $\mathrm{NaCl}$ after being etched with $\mathrm{HF}$ imaged at EHT of $2.55 \mathrm{kV}$, with a scale bar of $100 \mathrm{~nm}$, and imaged with the InLens detector. Top: 178.44 kx magnification. Bottom: 275 kx magnification. 
Stöber without $\mathrm{NaCl}$ after $\mathrm{HF}$ etch:

When $\mathrm{NaCl}$ was used as a heat sink during magnesiothermic reduction, then etched with HF, the Stöber nanoparticles appear amorphous and fused together as depicted in Figures 3.35, 3.26 , and 3.27 .

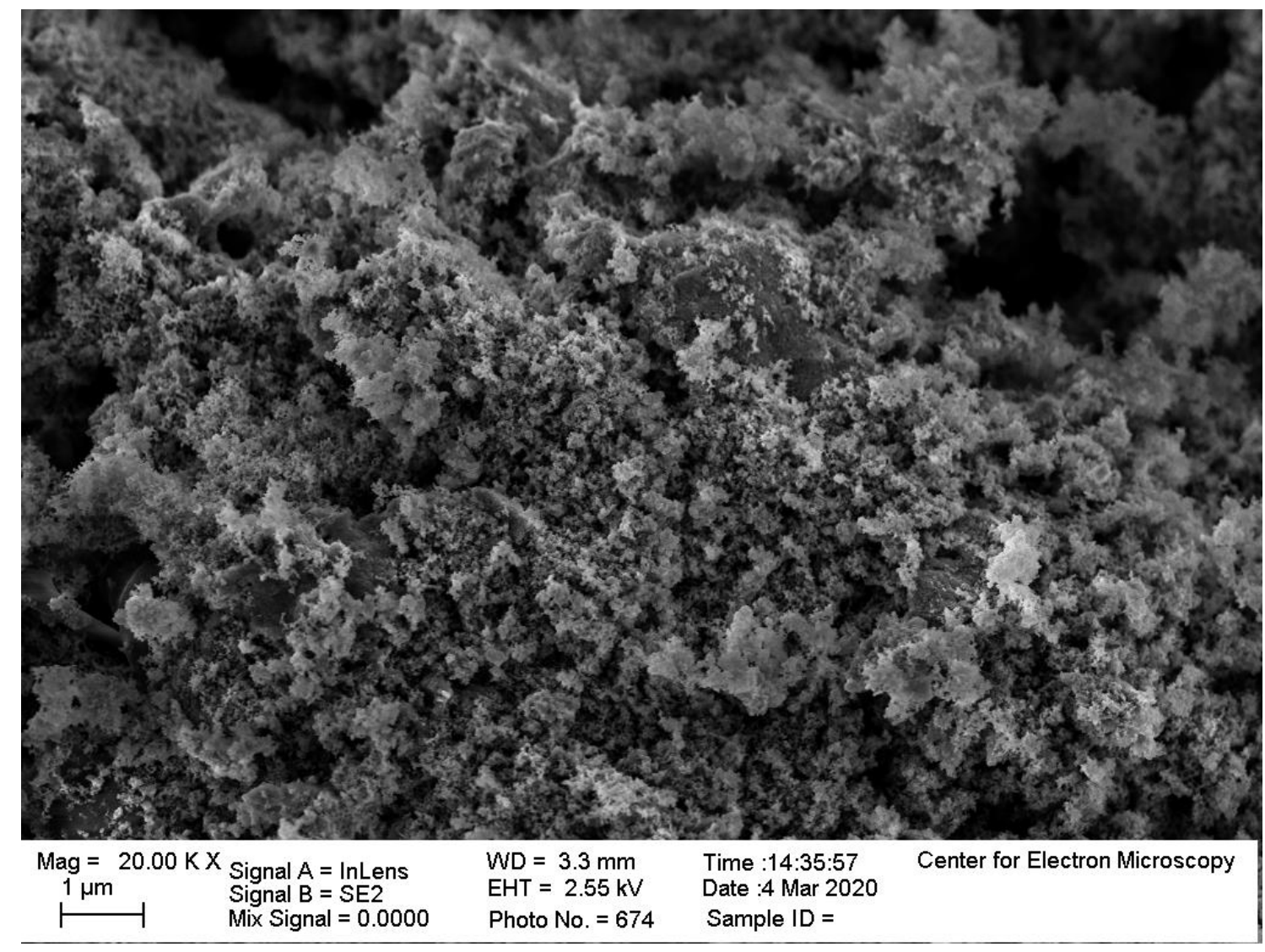

Figure 3.25. SEM micrograph of Stöber nanoparticles without $\mathrm{NaCl}$ after being etched with $\mathrm{HF}$ imaged at EHT of $2.55 \mathrm{kV}$ with the InLens detector, a scale bar of $1 \mathrm{um}$, and $20 \mathrm{kx}$ magnification. 


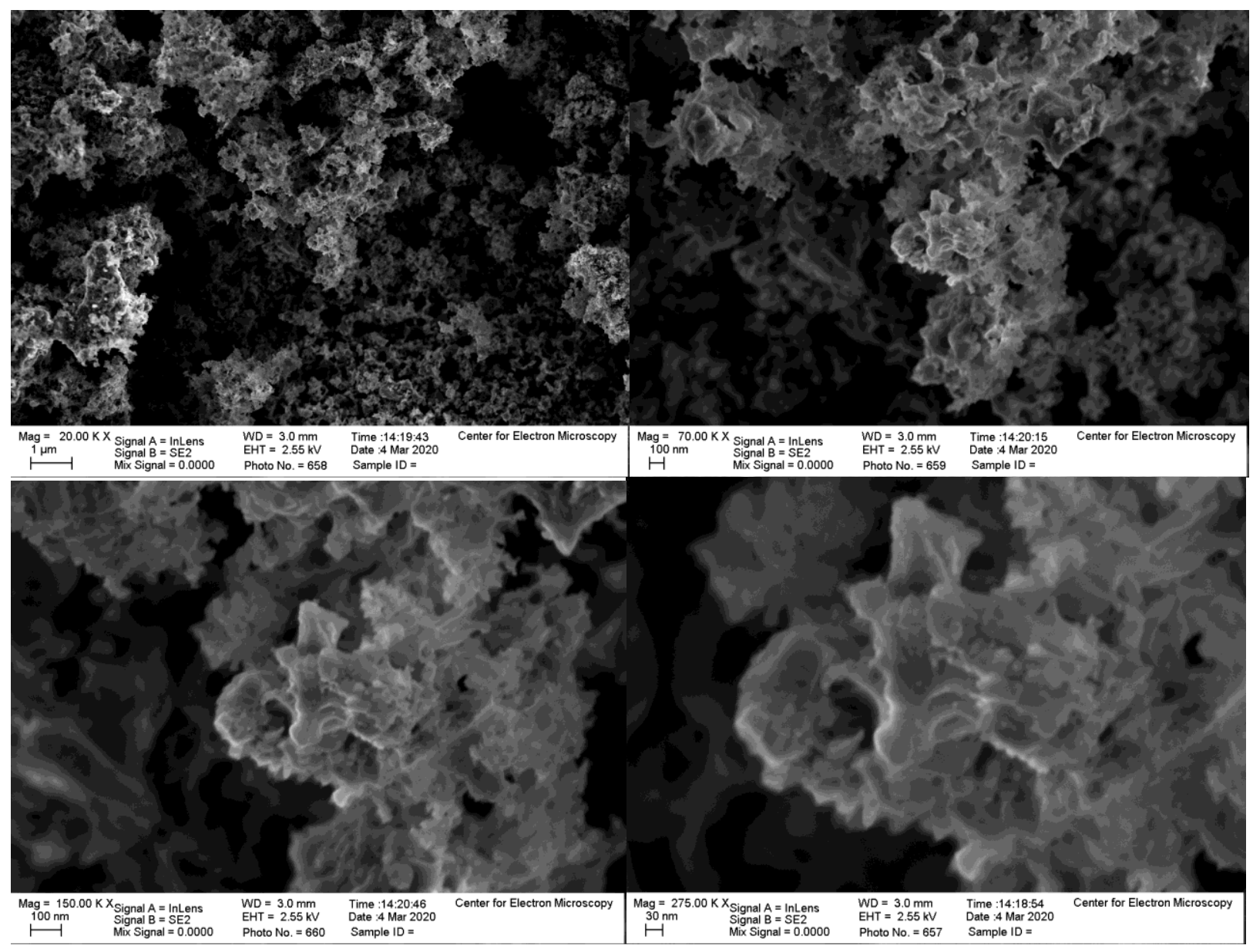

Figure 3.26. SEM micrograph of Stöber nanoparticles without $\mathrm{NaCl}$ after being etched with $\mathrm{HF}$ imaged at EHT of $2.55 \mathrm{kV}$ with the InLens detector at the same location. Top left: $20 \mathrm{kx}$ magnification and a scale bar of $1 \mathrm{um}$. Top right: $70 \mathrm{kx}$ magnification and a scale bar of $100 \mathrm{~nm}$. Bottom left: $150 \mathrm{kx}$ magnification and a scale bar of $100 \mathrm{~nm}$. Bottom right: $275 \mathrm{kx}$ magnification and a scale bar of $30 \mathrm{~nm}$. 


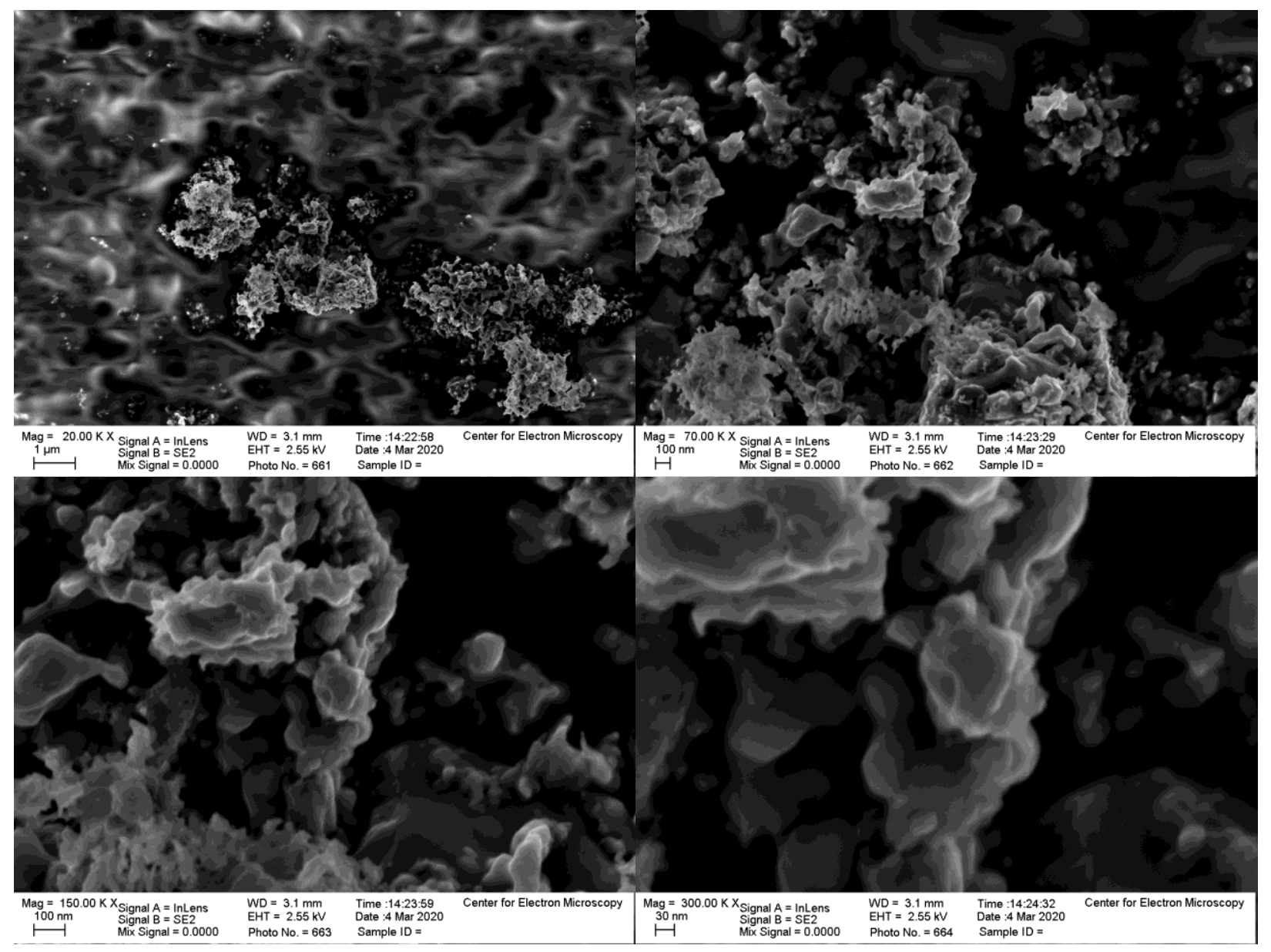

Figure 3.27. SEM micrograph of Stöber nanoparticles without $\mathrm{NaCl}$ after being etched with $\mathrm{HF}$ imaged at EHT of $2.55 \mathrm{kV}$ with the InLens detector at the same location. Top left: $20 \mathrm{kx}$ magnification and a scale bar of $1 \mathrm{um}$. Top right: $70 \mathrm{kx}$ magnification and a scale bar of $100 \mathrm{~nm}$. Bottom left: $150 \mathrm{kx}$ magnification and a scale bar of $100 \mathrm{~nm}$. Bottom right: $300 \mathrm{kx}$ magnification and a scale bar of $30 \mathrm{~nm}$.

In a different region of the sample, a honeycomb-like structure is visible, as imaged in Figures 3.28, 3.29, and 3.30. 

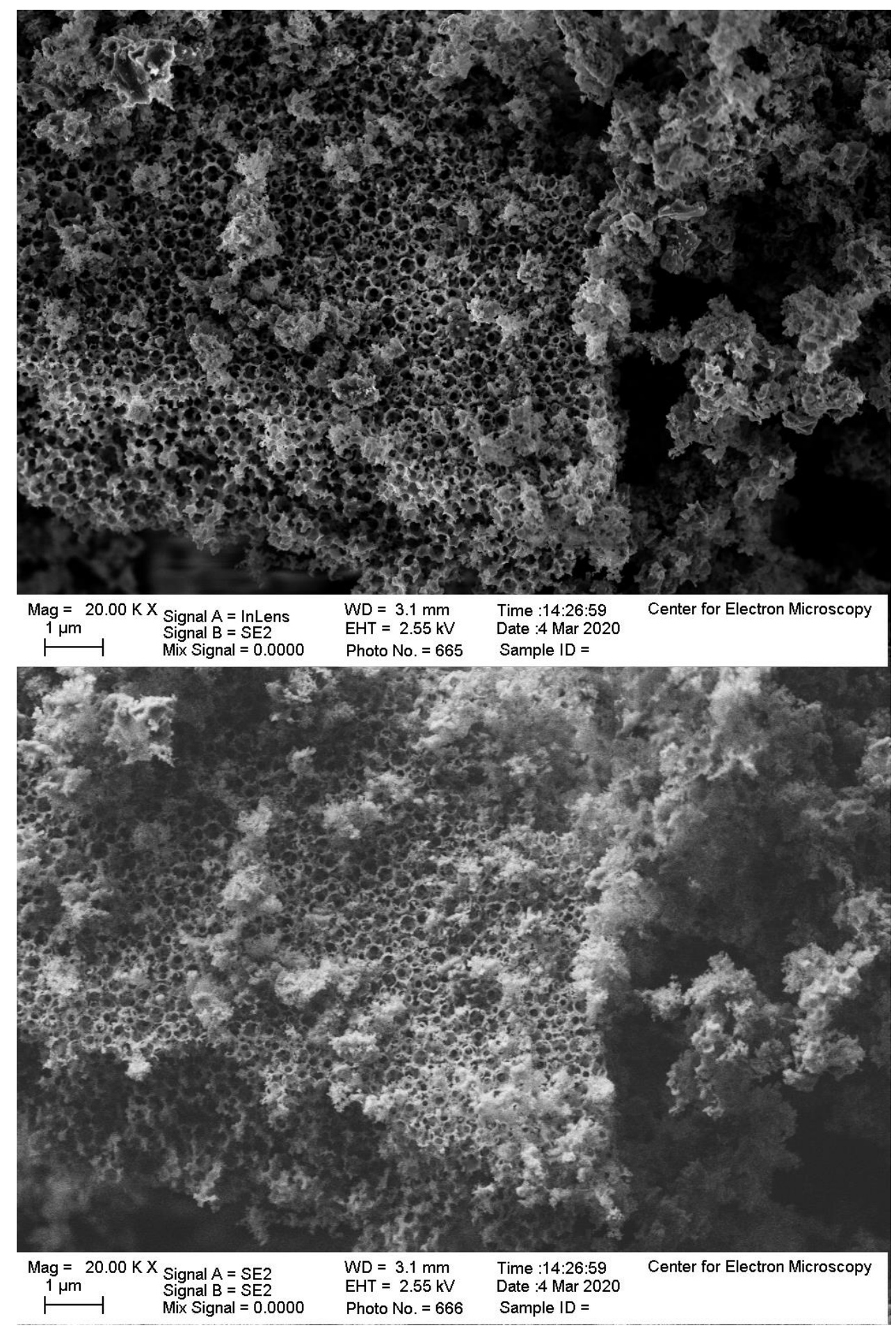

Figure 3.28. SEM micrograph of Stöber nanoparticles without $\mathrm{NaCl}$ after being etched with $\mathrm{HF}$ imaged at EHT of $2.55 \mathrm{kV}$ at the same location, $20 \mathrm{kx}$, and $1 \mathrm{um}$ scale bar. Top: InLens detector. Bottom: SE2 detector. 


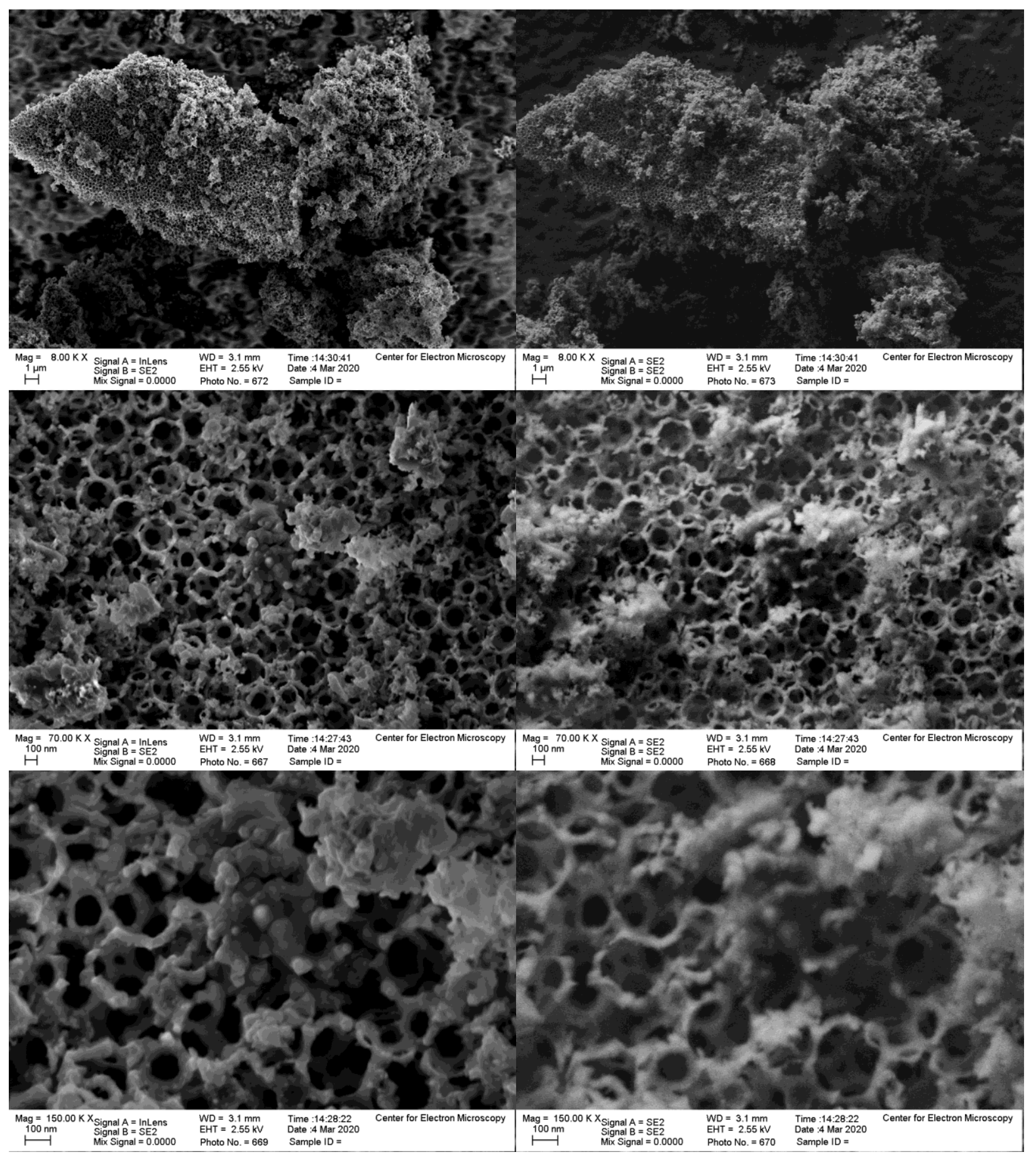

Figure 3.29. SEM micrograph of Stöber nanoparticles without $\mathrm{NaCl}$ after being etched with $\mathrm{HF}$ imaged at EHT of $2.55 \mathrm{kV}$ at the same location. All of the images on the left: InLens. All images on the right: SE2. Top images: $8 \mathrm{kx}$ magnification, $1 \mathrm{um}$ scale bar. The following images have a $100 \mathrm{~nm}$ scale bar. Middle images: 70 kx magnification. Bottom images: 150 kx magnification. 


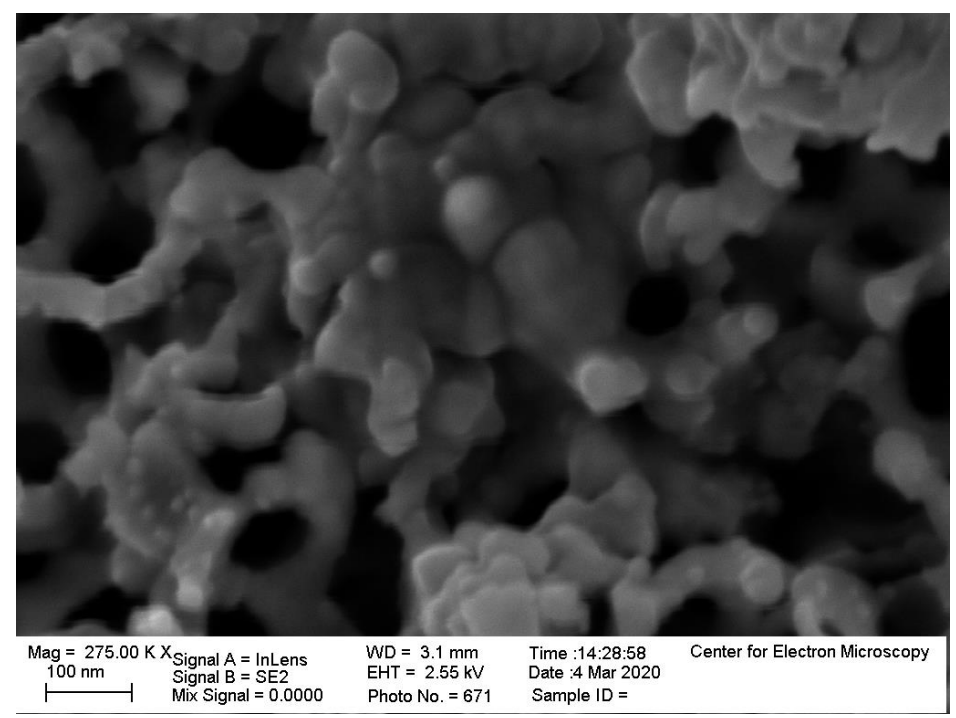

Figure 3.30. SEM micrograph of Stöber nanoparticles without $\mathrm{NaCl}$ after being etched with $\mathrm{HF}$ imaged at EHT of $2.55 \mathrm{kV}$ with the InLens detector, a $100 \mathrm{~nm}$ scale bar, and $275 \mathrm{kx}$ magnification.

Table 3.1 Summary of experimental results.

\begin{tabular}{|c|c|c|c|c|c|}
\hline & & Spherical & Fused & Amorphous & Honeycomb \\
\hline \multicolumn{6}{|c|}{ Starting Materials } \\
\hline 1 & Stöber & $\mathrm{x}$ & & & \\
\hline 2 & NanoXact & $\mathrm{x}$ & & & \\
\hline \multicolumn{6}{|c|}{ Magnesiothermic Reduction with $\mathrm{NaCl}$} \\
\hline 3 & Stöber & $\mathrm{x}$ & & $\mathrm{x}$ & \\
\hline 4 & NanoXact & $\mathrm{x}$ & & $\mathrm{x}$ & \\
\hline \multicolumn{6}{|c|}{ Magnesiothermic Reduction without $\mathrm{NaCl}$} \\
\hline 5 & Stöber & $\mathrm{x}$ & $\mathrm{x}$ & $\mathrm{x}$ & \\
\hline 6 & NanoXact & $\mathrm{x}$ & & $\mathrm{x}$ & \\
\hline \multicolumn{6}{|c|}{ Etching with HF } \\
\hline 7 & Stöber $(\mathrm{NaCl})$ & $\mathrm{x}$ & $\mathrm{x}$ & $\mathrm{x}$ & \\
\hline 8 & Stöber & & & & $\mathrm{x}$ \\
\hline 9 & NanoXact $(\mathrm{NaCl})$ & $\mathrm{x}$ & $\mathrm{x}$ & $\mathrm{x}$ & \\
\hline 10 & NanoXact & & $\mathrm{x}$ & $\mathrm{x}$ & \\
\hline
\end{tabular}

Table 3.1 displays the morphological differences of the nanoparticles throughout the experiment. 


\section{DISCUSSION}

The starting materials commercially available NanoXact and lab synthesized Stöber nanoparticles are both spherical. Significant observations include that there was retention of morphology on a nanoscale similar to starting material without using the HF etching process, regardless of the addition of $\mathrm{NaCl}$. $\mathrm{NaCl}$ used as a heat sink does cause a few spherical objects to appear. However, the magnesiothermic reduction does create many amorphous structures. The nanoparticles after the HF etching process are believed to be pure elemental silicon. The holes may be the location of silica that was etched away with HF, after HF etching morphology is retained/ragged. The Stöber particles without $\mathrm{NaCl}$ as a heat sink created honeycomb-like structures with some impurities.

The InLens has a higher S:N ratio than the SE2 detector, and the SE2 detector portrays enhanced topography in comparison to the InLens detector. There were difficulties in imaging the NanoXact nanoparticles with $\mathrm{NaCl}$.

\section{CONCLUSION}

For both the commercially available NanoXact and laboratory synthesized Stöber nanoparticles prior to magnesiothermic reduction, the nanoparticles appear solid and spherical. Following magnesiothermic reduction, morphology is preserved with or without $\mathrm{NaCl}$ as a heat sink. However, Stöber nanoparticles without $\mathrm{NaCl}$ as a heat sink also appeared to fuse together while retaining some morphology from the starting material. Following the HF etching process, NanoXact and Stöber nanoparticles with $\mathrm{NaCl}$ were amorphous and fused together. The NanoXact nanoparticles without $\mathrm{NaCl}$ that were etched with $\mathrm{HF}$ appear amorphous, but due to the low magnification limitation, it is difficult to deduce if the nanoparticles are fused or partially 
spherical. The Stöber nanoparticles without $\mathrm{NaCl}$ after the $\mathrm{HF}$ etch have a honeycomb-like structure with some impurities. Both NanoXact and Stöber nanoparticles after magnesiothermic reduction with and without $\mathrm{NaCl}$ as a heat sink retained a spherical morphology similar to the starting material. Once the HF etching process is completed, it is challenging to retain morphology. A majority of the experiment produced results we were not looking for. However, if the honeycomb structure of the Stöber nanoparticles without $\mathrm{NaCl}$ after $\mathrm{HF}$ etch could be replicated without impurities, they could be manufactured to increase surface area for silicon anodes on batteries.

\section{FUTURE WORK}

Since the Stöber process should be size, shape, and morphology preserving, future experiments could include varying reaction conditions that produce solid crystalline cores while maintaining spherical shape. Altering SEM parameters may increase resolution, or higher resolution can be achieved using a more powerful SEM. The goal would be to vary reaction conditions that produce solid crystalline cores while maintaining spherical shape. It would be interesting to determine similarities to the starting material, and whether the nanoparticles were actually solid or porous. Since there was an XRD mismatch, SEM would be used to determine the mismatch. Use of PELCO Tabs 12mm OD instead of carbon tape to image nanoparticles may increase the quality of the images deterring image distortion. EDX could be used to determine if $\mathrm{SiO} 2$ is a byproduct following magnesiothermic reduction with and without $\mathrm{NaCl}$ after magnesiothermic reduction; additionally, it can be used for the same reason following the HF etch. Silicon is more conductive than silica, so it may be easier to image Si due to a reduction in charging effects. Another question to consider would be how and why does surface area increase in silicon after magnesiothermic reduction? 


\section{ACKNOWLDGEMENTS}

Dr. Goforth, James Barnes, The Goforth Lab, CEMN, Greg Baty, Dr. Chen, and The PSU Physics Department. 


\section{REFERENCES}

${ }^{1}$ Entwistle, J., Rennie, A., \&amp; Patwardhan, S. (2018). A review of magnesiothermic reduction of silica to porous silicon for lithium-ion battery applications and beyond. Journal of Materials Chemistry A, 6(38), 18344-18356. https://doi.org/10.1039/c8ta06370b

${ }^{2}$ Plumeré, N., Ruff, A., Speiser, B., Feldmann, V., Mayer, H. A. (2012). Stöber silica particles as basis for redox modifications: Particle shape, size, polydispersity, and porosity. Journal of Colloid and Interface Science, 368(1), 208219.https://doi.org/10.1016/j.jcis.2011.10.070

${ }^{3}$ Lai, Y., Thompson, J. R., \&amp; Dasog, M. (2018). Metallothermic Reduction of Silica Nanoparticles to Porous Silicon for Drug Delivery Using New and Existing Reductants. Chemistry - A European Journal, 24(31), 7913-7920. doi:10.1002/chem.201705818

${ }^{4}$ Hadipour Moghaddam, S. P., Yazdimamaghani, M., \& Ghandehari, H. (2018). Glutathionesensitive hollow mesoporous silica nanoparticles for controlled drug delivery. Journal of controlled release : official journal of the Controlled Release Society, 282, 62-75. https://doi.org/10.1016/j.jconrel.2018.04.032

${ }^{5}$ Julian, M. M. (2015). Foundations of crystallography with computer applications. Boca Raton: Taylor \& Francis.

${ }^{6}$ Goldstein, J. I., Newbury, D. E., Michael, J. R., Ritchie, N. W., Scott, J. H., \& Joy, D. C. (2018). Scanning Electron Microscopy and X-Ray Microanalysis. New York, NY: Springer New York.

${ }^{7}$ nanoComposix. Silica Surface. nanoComposix. https://nanocomposix.com/pages/silica-surface. 
${ }^{8}$ Dasog, M., Yang, Z., \&amp; Veinot, J. G. (2012). Size-controlled solid state synthesis of luminescent silicon nanocrystals using Stöber silica particles. CrystEngComm, 14(22), 7576. doi:10.1039/c2ce25950h

${ }^{9}$ Rapp, R.A., Ezis, A. \& Yurek, G.J. Displacement reactions in the solid state. MT 4, 1283-1292 (1973). https://doi.org/10.1007/BF02644523 